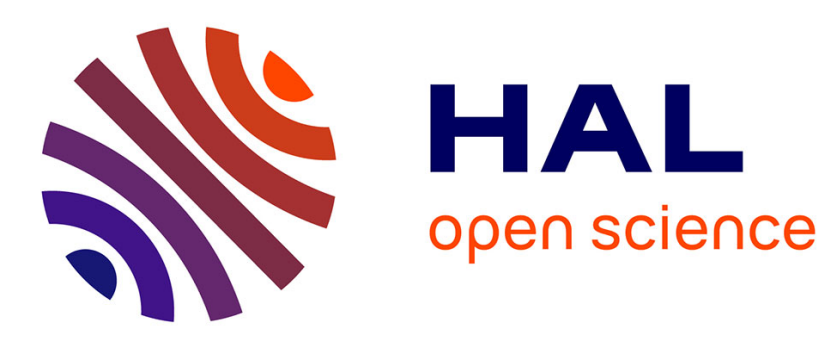

\title{
Modeling and Control of mini UAV
}

Gerardo Ramon Flores Colunga, A. Guerrero, Juan Antonio Escareño, Rogelio Lozano

\section{To cite this version:}

Gerardo Ramon Flores Colunga, A. Guerrero, Juan Antonio Escareño, Rogelio Lozano. Modeling and Control of mini UAV. Josep Guerrero (Editor), Rogelio Lozano (Editor). Flight Formation Control, Wiley, pp.99-134, 2012, 978-1-84821-323-4. hal-00923127

\section{HAL Id: hal-00923127 https://hal.science/hal-00923127}

Submitted on 2 Jan 2014

HAL is a multi-disciplinary open access archive for the deposit and dissemination of scientific research documents, whether they are published or not. The documents may come from teaching and research institutions in France or abroad, or from public or private research centers.
L'archive ouverte pluridisciplinaire HAL, est destinée au dépôt et à la diffusion de documents scientifiques de niveau recherche, publiés ou non, émanant des établissements d'enseignement et de recherche français ou étrangers, des laboratoires publics ou privés. 


\title{
UAV Flight Formation Control
}

\author{
Jose Alfredo GUERRERO
}

Rogelio LOZANO

Version 0.5, 


\section{Contents}

Chapter 1. Modeling and Control of Mini UAV . . . . . . . . . . . . . . 1

G. Flores , J.A. Guerrero, J. Escareno, R. Lozano

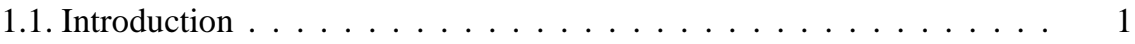

1.2. The Quad-Rotor . . . . . . . . . . . . . . . . . . . 2

1.2.1. Dynamical Model: Euler-Lagrange Approach . . . . . . . . . 4

1.3. Control of a Mini Tail-Sitter . . . . . . . . . . . . . . . . . . . . 8

1.3.1. Linear Control Strategy . . . . . . . . . . . . . . . . . . . . . 9

1.3.1.1. Roll control . . . . . . . . . . . . . . . . . 10

1.3.1.2. Pitch control . . . . . . . . . . . . . . . . . . . . 13

1.3.1.3. Yaw control . . . . . . . . . . . . . . . . . . . . . . . . . . 14

1.3.1.4. Simulation Results . . . . . . . . . . . . . . . . . . . . . . . . . . . . .

1.3.1.5. Experimental Results . . . . . . . . . . . . . . 16

1.3.2. Robust Control Considering Parametric Uncertainty . . . . . . . . . . 17

1.3.2.1. Pitch Subsystem . . . . . . . . . . . . . . . . . . . . . . . . . 18

1.3.2.2. Yaw Subsystem . . . . . . . . . . . . . . . . . . . . . . . . . . . . . 19

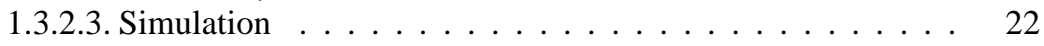

1.3.3. Nonlinear Control based on Nested Saturations . . . . . . . . . . . . . 23

1.3.3.1. Equations of Translational Motion . . . . . . . . . . . . 25

1.3.3.2. Simulation Results . . . . . . . . . . . . . . . . . . . . . . . . . . . 30

1.4. Quad-Tilting Rotor Convertible MAV . . . . . . . . . . . . . . . . . . . . . . . . . . 30

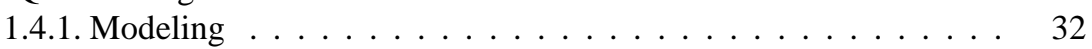

1.4.2. Transition . . . . . . . . . . . . . . . . 39

1.4.3. Control strategy for hover flight mode . . . . . . . . . . . . . . . 41

1.4.4. Control strategy for forward flight mode . . . . . . . . . . . 43

1.4.5. Simulation results . . . . . . . . . . . . . . . . . . . 46

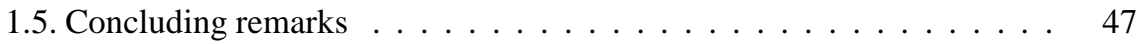

1.6. Bibliography .......................... 48 


\section{Chapter 1}

\section{Modeling and Control of Mini UAV}

This Chapter deals with the modeling and control of different configurations of the UAVs, and is organized as follows. Section 1.2 gives a general overview of the quad-rotor aerial vehicle and its operation principle. The modeling is presented using the Euler-Lagrange approach. Sections 1.3 and 1.4 deal with the Hybrid or Convertible MAVs, combining the advantages of horizontal and vertical flight. Different approaches for non-linear control are presented using the Lyapunov theory. Finally, some concluding remarks are presented in Section 1.5.

\subsection{Introduction}

The applications of mini Unmanned Aerial Vehicles (UAVs) comprise both military and civilian, though the latter has had a lower development rate. The use of aerial robots, specially miniature (mini and micro) UAVs (MAVs), has enhanced activities such as surveillance of sensible areas (borders, harbors, prisons), wildlife study, natural disasters assessment, traffic surveillance, pollution monitoring, just to mention a few. However, there are missions whose scope is beyond the capabilities of conventional small UAVs designs since they require not only longer flight endurance but also hovering/VTOL capabilities. Besides the commonly used aerial vehicles, the Hybrid or Convertible MAVs, have been gaining popularity recently. By marrying the takeoff and landing capabilities of the helicopter with the forward flight efficiencies of fixed-wing aircraft, the Convertible UAV promise a unique blend of capabilities at lower cost than other UAV configurations. Two kinds of Convertible UAV vehicles are discussed: the Bidule mAV and the Quad-plane in section 1.3 and 1.4 respectively.

Chapter written by G. Flores and J.A. Guerrero and J. Escareno and R. Lozano. 
The complete dynamics of these kind of vehicles, taking into account aero-elastic effects, flexibility of the wings, internal dynamics of the engine and the whole set of changing variables are quite complex and somewhat unmanageable for the purposes of control. Therefore, it is interesting to consider a simplified model of an aircraft formed by a minimum number of states and inputs, but retaining the main features that must be considered when designing control laws for a real aircraft.

The development of the simplified model of the common Quadrotor will be presented and it will be used throughout the entire book.

\subsection{The Quad-Rotor}

The quad-rotor mini-rotorcraft is controlled by the angular speeds of four electric motors as shown in Figure 1.1. Each motor produces a thrust and a torque, whose combination generates the main thrust, the yaw torque, the pitch torque, and the roll torque acting on the quad-rotor. Conventional helicopters modify the lift force by

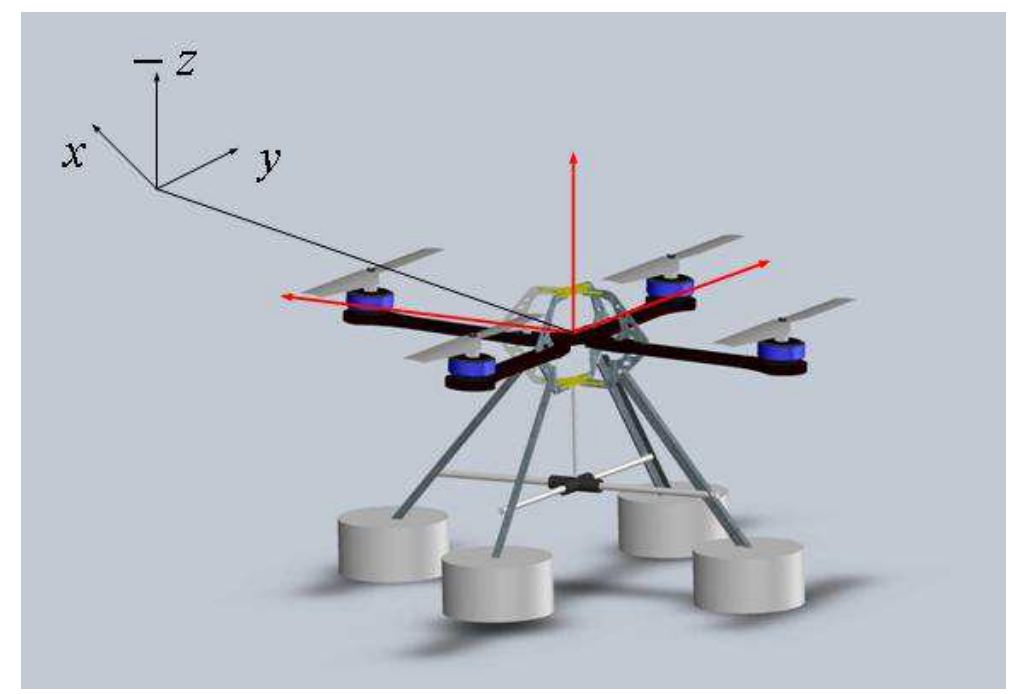

Figure 1.1: The quad-rotor in an inertial frame. $f_{1}, f_{2}, f_{3}, f_{4}$ represent the thrust of each motor, $\psi, \theta$ and $\phi$ represent the Euler angles, and $u$ is the main thrust.

varying the collective pitch. Such aerial vehicles use a mechanical device known as swashplate. This system interconnects servomechanisms and blade pitch links in order to change the rotor blades pitch angle in a cyclic manner, so as to obtain the pitch and roll control torques of the vehicle. In contrast, the quad-rotor does not have a swashplate and has constant pitch blades. Therefore, in a quad-rotor we can only 
vary the angular speed of each one of the four rotors to obtain the pitch and roll control torques.

From Figure 1.1 it can be observed that the motor $M_{i}$ (for $i=1, \ldots, 4$ ) produces the force $f_{i}$, which is proportional to the square of the angular speed, that is $f_{i}=k w_{i}^{2}$. Given that the quad-rotor's motors can only turn in a fixed direction, the produced force $f_{i}$ is always positive. The front $\left(M_{1}\right)$ and the rear $\left(M_{3}\right)$ motors rotate counter-clockwise, while the left $\left(M_{2}\right)$ and right $\left(M_{4}\right)$ motors rotate clockwise. With this arrangement, gyroscopic effects and aerodynamic torques tend to cancel in trimmed flight. The main thrust $u$ is the sum of individual thrusts of each motor. The pitch torque is a function of the difference $f_{1}-f_{3}$, the roll torque is a function of $f_{2}-f_{4}$, and the yaw torque is the sum $\tau_{M_{1}}+\tau_{M_{2}}+\tau_{M_{3}}+\tau_{M_{4}}$, where $\tau_{M_{i}}$ is the reaction torque of motor $i$ due to shaft acceleration and blades drag. The motor torque is opposed by an aerodynamic drag $\tau_{\text {drag }}$, such that

$$
I_{\text {rot }} \dot{\omega}=\tau_{M_{i}}-\tau_{\text {drag }}
$$

where $I_{\text {rot }}$ is the moment of inertia of a rotor around its axis. The aerodynamic drag is defined as

$$
\tau_{\text {drag }}=\frac{1}{2} \rho A v^{2}
$$

where $\rho$ is the air density, the frontal area of the moving shape is defined by $A$, and $v$ is its velocity relative to the air. In magnitude, the angular velocity $\omega$ is equal to the linear velocity $v$ divided by the radius of rotation $r$

$$
\omega=\frac{v}{r}
$$

The aerodynamic drag can be rewritten as

$$
\tau_{\text {drag }}=k_{\text {drag }} \omega^{2}
$$

where $k_{d r a g}>0$ is a constant depending on the air density, the radius, the shape of the blade and other factors. For quasi-stationary manoeuvres, $\omega$ is constant, then

$$
\tau_{M_{i}}=\tau_{d r a g}
$$

Forward pitch motion is obtained by increasing the speed of the rear motor $M_{3}$ while reducing the speed of the front motor $M_{1}$. similarly, roll motion is obtained using the left and right motors. Yaw motion is obtained by increasing the torque of the front and rear motors ( $\tau_{M 1}$ and $\tau_{M 3}$ respectively) while decreasing the torque of the lateral motors ( $\tau_{M 2}$ and $\tau_{M 4}$ respectively). Such motions can be accomplished while maintaining the total thrust constant. The quad-rotor model is obtained by representing the aircraft as a solid body evolving in a three dimensional space and subject to the main thrust and three torques: pitch, roll and yaw. 


\subsubsection{Dynamical Model: Euler-Lagrange Approach}

Let the generalized coordinates of the rotorcraft be expressed by

$$
\boldsymbol{q}=(x, y, z, \psi, \theta, \phi) \in \mathbb{R}^{6}
$$

where $\boldsymbol{\xi}=(x, y, z) \in \mathbb{R}^{3}$ denotes the position vector of the center of mass of the quadrotor relative to a fixed inertial frame $\mathcal{I}$. The rotorcraft's Euler angles (the orientation of the rotorcraft) are expressed by $\boldsymbol{\eta}=(\psi, \theta, \phi) \in \mathbb{R}^{3}, \psi$ is the yaw angle around the $z$-axis, $\theta$ is the pitch angle around the $y$-axis and $\phi$ is the roll angle around the $x$-axis (see [ETK 96]).An illustration of the generalized coordinates of the rotorcraft is shown in Figure ??. Define the Lagrangian

$$
L(\boldsymbol{q}, \dot{\boldsymbol{q}})=T_{\text {trans }}+T_{\text {rot }}-U
$$

where $T_{\text {trans }}=\frac{m}{2} \dot{\boldsymbol{\xi}}^{T} \dot{\boldsymbol{\xi}}$ is the translational kinetic energy, $T_{\text {rot }}=\frac{1}{2} \boldsymbol{\Omega}^{T} \boldsymbol{I} \boldsymbol{\Omega}$ is the rotational kinetic energy, $U=m g z$ is the potential energy of the rotorcraft, $z$ is the rotorcraft altitude, $m$ denotes the mass of the quad-rotor, $\Omega$ is the vector of the angular velocity, $\boldsymbol{I}$ is the inertia matrix and $g$ is the acceleration due to gravity. The angular velocity vector $\boldsymbol{\omega}$ resolved in the body fixed frame is related to the generalized velocities $\dot{\eta}$ (in the region where the Euler angles are valid) by means of the standard kinematic relationship [GOL 83]

$$
\Omega=W_{\eta} \dot{\eta}
$$

where

$$
\boldsymbol{W}_{\boldsymbol{\eta}}=\left[\begin{array}{ccc}
-\sin \theta & 0 & 1 \\
\cos \theta \sin \phi & \cos \phi & 0 \\
\cos \theta \cos \phi & -\sin \phi & 0
\end{array}\right]
$$

then

$$
\boldsymbol{\Omega}=\left[\begin{array}{c}
\dot{\phi}-\dot{\psi} \sin \theta \\
\dot{\theta} \cos \phi+\dot{\psi} \cos \theta \sin \phi \\
\dot{\psi} \cos \theta \cos \phi-\dot{\theta} \sin \phi
\end{array}\right]
$$


Define

$$
\boldsymbol{J}=\boldsymbol{J}(\boldsymbol{\eta})=\boldsymbol{W}_{\boldsymbol{\eta}}^{T} \boldsymbol{I} \boldsymbol{W}_{\boldsymbol{\eta}}
$$

where

$$
\boldsymbol{I}=\left[\begin{array}{ccc}
I_{x x} & 0 & 0 \\
0 & I_{y y} & 0 \\
0 & 0 & I_{z z}
\end{array}\right]
$$

so that

$$
T_{\text {rot }}=\frac{1}{2} \dot{\boldsymbol{\eta}}^{T} \boldsymbol{J} \dot{\boldsymbol{\eta}}
$$

Thus, the matrix $\boldsymbol{J}=\boldsymbol{J}(\boldsymbol{\eta})$ acts as the inertia matrix for the full rotational kinetic energy of the quad-rotor, expressed directly in terms of the generalized coordinates $\boldsymbol{\eta}$.

The model of the full rotorcraft dynamics is obtained from Euler-Lagrange equations with external generalized forces

$$
\frac{d}{d t}\left(\frac{\partial \mathcal{L}}{\partial \dot{\boldsymbol{q}}}\right)-\frac{\partial \mathcal{L}}{\partial \boldsymbol{q}}=\left[\begin{array}{c}
\boldsymbol{F}_{\boldsymbol{\xi}} \\
\boldsymbol{\tau}
\end{array}\right]
$$

where $\boldsymbol{F}_{\boldsymbol{\xi}}=\boldsymbol{R} \hat{\boldsymbol{F}} \in \mathbb{R}^{3}$ is the translational force applied to the rotorcraft due to main thrust, $\boldsymbol{\tau} \in \mathbb{R}^{3}$ represents the yaw, pitch and roll moments and $\boldsymbol{R}$ denotes the rotational matrix. $\boldsymbol{R}(\psi, \theta, \phi) \in \boldsymbol{S} \boldsymbol{O}(3)$ represents the orientation of the aircraft relative to a fixed inertial frame

$$
\boldsymbol{R}=\left[\begin{array}{ccc}
c_{\theta} c_{\psi} & c_{\psi} s_{\theta} s_{\phi}-c_{\phi} s_{\psi} & s_{\phi} s_{\psi}+c_{\phi} c_{\psi} s_{\theta} \\
c_{\theta} s_{\psi} & c_{\phi} c_{\psi}+s_{\theta} s_{\phi} s_{\psi} & c_{\phi} s_{\theta} s_{\psi}-c_{\psi} s_{\phi} \\
-s_{\theta} & c_{\theta} s_{\phi} & c_{\theta} c_{\phi}
\end{array}\right]
$$

where $c_{\theta}$ stands for $\cos \theta$ and $s_{\theta}$ for $\sin \theta$. From Figure 1.1, it follows that

$$
\hat{\boldsymbol{F}}=\left[\begin{array}{l}
0 \\
0 \\
u
\end{array}\right]
$$


where $u$ is the main thrust directed out of the bottom of the aircraft and expressed as

$$
u=\sum_{i=1}^{4} f_{i}
$$

and, for $i=1, \ldots, 4, f_{i}$ is the force produced by motor $M_{i}$, as shown in Figure 1.1. Typically $f_{i}=k \omega_{i}^{2}$, where $k_{i}$ is a constant and $\omega_{i}$ is the angular speed of the $i$-th motor. The generalized torques are thus

$$
\boldsymbol{\tau}=\left[\begin{array}{c}
\tau_{\psi} \\
\tau_{\theta} \\
\tau_{\phi}
\end{array}\right] \triangleq\left[\begin{array}{c}
\sum_{i=1}^{4} \tau_{M_{i}} \\
\left.\left(f_{2}-f_{4}\right)\right) \ell \\
\left.\left(f_{3}-f_{1}\right)\right) \ell
\end{array}\right]
$$

where $\ell$ is the distance between the motors and the center of gravity, and $\tau_{M_{i}}$ is the moment produced by motor $M_{i}$, for $i=1, \ldots, 4$, around the center of gravity of the aircraft.

Since the Lagrangian contains no cross terms in the kinematic energy combining $\dot{\xi}$ with $\dot{\eta}$, the Euler-lagrange equation can pe partitioned into dynamics for $\boldsymbol{\xi}$ coordinates and $\boldsymbol{\eta}$ coordinates. The Euler-Lagrange equation for the translational motion is

$$
\frac{d}{d t}\left[\frac{\partial L_{\text {trans }}}{\partial \dot{\boldsymbol{\xi}}}\right]-\frac{\partial L_{\text {trans }}}{\partial \boldsymbol{\xi}}=\boldsymbol{F}_{\boldsymbol{\xi}}
$$

then

$$
m \ddot{\boldsymbol{\xi}}+m g \boldsymbol{E}_{\boldsymbol{z}}=\boldsymbol{F}_{\boldsymbol{\xi}}
$$

As for the $\boldsymbol{\eta}$ coordinates it can be written

$$
\frac{d}{d t}\left[\frac{\partial L_{r o t}}{\partial \dot{\boldsymbol{\eta}}}\right]-\frac{\partial L_{r o t}}{\partial \boldsymbol{\eta}}=\boldsymbol{\tau}
$$

or

$$
\frac{d}{d t}\left[\dot{\boldsymbol{\eta}}^{T} \boldsymbol{J} \frac{\partial \dot{\boldsymbol{\eta}}}{\partial \boldsymbol{\eta}}\right]-\frac{1}{2} \frac{\partial}{\partial \boldsymbol{\eta}}\left(\dot{\boldsymbol{\eta}}^{T} \boldsymbol{J} \dot{\boldsymbol{\eta}}\right)=\boldsymbol{\tau}
$$


Thus one obtains

$$
\boldsymbol{J} \ddot{\boldsymbol{\eta}}+\dot{\boldsymbol{J}} \dot{\boldsymbol{\eta}}-\frac{1}{2} \frac{\partial}{\partial \boldsymbol{\eta}}\left(\dot{\boldsymbol{\eta}}^{T} \boldsymbol{J} \dot{\boldsymbol{\eta}}\right)
$$

Defining the Coriolis-Centripetal vector

$$
\overline{\boldsymbol{V}}(\boldsymbol{\eta}, \dot{\boldsymbol{\eta}})=\dot{\boldsymbol{J}} \dot{\boldsymbol{\eta}}-\frac{1}{2} \frac{\partial}{\partial \boldsymbol{\eta}}\left(\dot{\boldsymbol{\eta}}^{T} \boldsymbol{J} \dot{\boldsymbol{\eta}}\right)
$$

one writes

$$
\boldsymbol{J} \ddot{\boldsymbol{\eta}}+\overline{\boldsymbol{V}}(\boldsymbol{\eta}, \dot{\boldsymbol{\eta}})=\boldsymbol{\tau}
$$

but $\overline{\boldsymbol{V}}(\boldsymbol{\eta}, \dot{\boldsymbol{\eta}})$ can be expressed as

$$
\begin{aligned}
\overline{\boldsymbol{V}}(\boldsymbol{\eta}, \dot{\boldsymbol{\eta}}) & =\left(\dot{\boldsymbol{J}}-\frac{1}{2} \frac{\partial}{\partial \boldsymbol{\eta}}\left(\dot{\boldsymbol{\eta}}^{T} \boldsymbol{J}\right)\right) \dot{\boldsymbol{\eta}} \\
& =\boldsymbol{C}(\boldsymbol{\eta}, \dot{\boldsymbol{\eta}}) \dot{\boldsymbol{\eta}}
\end{aligned}
$$

where $\boldsymbol{C}(\boldsymbol{\eta}, \dot{\boldsymbol{\eta}})$ is referred to as the Coriolis terms and contains the gyroscopic and centrifugal terms associated with the $\boldsymbol{\eta}$ dependence of $\boldsymbol{J}$. This yields

$$
\begin{aligned}
m \ddot{\boldsymbol{\xi}}+m g \boldsymbol{E}_{\boldsymbol{z}} & =\boldsymbol{F}_{\boldsymbol{\xi}} \\
\boldsymbol{J} \ddot{\boldsymbol{\eta}} & =\boldsymbol{\tau}-\boldsymbol{C}(\boldsymbol{\eta}, \dot{\boldsymbol{\eta}}) \dot{\boldsymbol{\eta}}
\end{aligned}
$$

To simplify lets make

$$
\tilde{\boldsymbol{\tau}}=\left(\begin{array}{c}
\tilde{\tau}_{\psi} \\
\tilde{\tau}_{\theta} \\
\tilde{\tau}_{\phi}
\end{array}\right)=\boldsymbol{J}^{-1}(\boldsymbol{\tau}-\boldsymbol{C}(\boldsymbol{\eta}, \dot{\boldsymbol{\eta}}) \dot{\boldsymbol{\eta}})
$$


Finally one obtains

$$
\begin{aligned}
m \ddot{x} & =u(\sin \phi \sin \psi+\cos \phi \cos \psi \sin \theta) \\
m \ddot{y} & =u(\cos \phi \sin \theta \sin \psi-\cos \psi \sin \phi) \\
m \ddot{z} & =u \cos \theta \cos \phi-m g \\
\ddot{\psi} & =\tilde{\tau}_{\psi} \\
\ddot{\theta} & =\tilde{\tau}_{\theta} \\
\ddot{\phi} & =\tilde{\tau}_{\phi}
\end{aligned}
$$

where $x$ and $y$ are coordinates in the horizontal plane, $z$ is the vertical position, and $\tilde{\tau}_{\psi}$, $\tilde{\tau}_{\theta}$ and $\tilde{\tau}_{\phi}$ are the yawing moment, pitching moment and rolling moment, respectively, which are related to the generalized torques $\tau_{\psi}, \tau_{\theta}, \tau_{\phi}$.

\subsection{Control of a Mini Tail-Sitter}

Tail-sitter vehicles represent a configuration of aircraft that remains relatively unexplored. Tail-sitters have more operational flexibility than conventional UAVs because a vertical airframe attitude is adopted during take-off and landing, while maintaining a horizontal airframe attitude during cruise just like conventional airplanes. Tail-sitters have not been as widely adopted as an aircraft configuration due to complex flight dynamics in the hover mode, making them typically very difficult to control.

The Convair XF-Y1 and Lockheed XF-V1 were examples of experimental Tailsitters aircraft in the 1950s, but they were unsuccessful mostly due to the problem caused by the awkward position of pilot required during the vertical flight phases, which would not be relevant for UAVs. In the 1990s Boeing presented its tail-sitter Heliwing UAV with a flight controller using cyclic-pitch rotor control for its vertical flight phases [CAS 05], while more recently in [STO 02a], the University of Sydney's T-Wing UAV has an autopilot which uses control surfaces in the slipstream of fixedpitch propellers for control in its vertical flight phases.

In recent years, interest in Vertical Take-Off and Landing (VTOL) mini Air vehicles (mAVs) have increased significantly due to a desire to operate UAVs in an urban environment. Many concepts have been proposed globally [BLY 06]. The Bidule mAV was developed at the University of Sydney to explore design issues related to small flight platforms [SPO 01]. The latest version, the Bidule CSyRex, is a joint project between the University of Sydney and the University of Technology of Compiègne to develop a VTOL variant of the Bidule. The vertical flight schematic of 
this VTOL vehicle is shown in Figure ??, which is basically a fixed wing tailless aircraft with two propellers. In hover mode, the altitude is controlled with the collective thrust, this means, the lift force is generated increasing the lift force produced by the propellers. The pitch attitude angular displacement is achieved by moving the elevons in the same direction. The vertical yaw-attitude angular displacement is achieved through moving the elevons in opposing direction. The vertical roll-attitude angular displacement is controlled by changing the pitch angle of the Variable Pitch Propeller (VPP). Typically, mAVs, such as the Planar Vertical Takeoff and Landing (PVTOL) platforms [CAS 05] modify the speed of the DC electric motors to effect altitude and attitude control. But when, brushless electric AC motors are used control responses have been too slow due to the time delay produced by the available speed controllers, leading to problems utilizing motor speed for roll control. VPP is thus being investigated as a potential solution, increasing the control response. This allows to implement a simple flight controller without considering the time-delay in actuators.

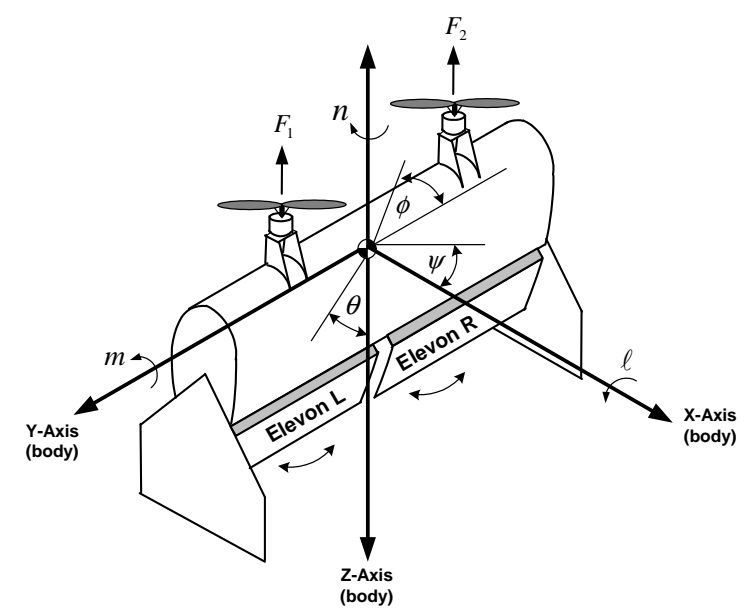

Figure 1.2: Vehicle schematic for vertical flight mode of Bidule

\subsubsection{Linear Control Strategy}

In this section, the main purpose is to control the attitude of the VTOL in hover flight. Therefore only the kinematic and moment equations will be used to obtain three decoupled attitude system for the pitch, roll and yaw angular position. The vehicle main wing has a profile NACA0008. 
Three decoupled stability augmentation control systems for the roll, the pitch, and the yaw positions of the vehicle in hover flight are developed. These subsystems will be obtained using only the kinematics and moment equations from the general model. Several aerodynamic factors will be taken into account to obtain the transfer function that represents the dynamic of each system.

\subsubsection{Roll control}

To obtain the roll control system, it is assumed that the pitch and yaw rates are zero. Then, the vehicle can be analyzed in a similar manner to a PVTOL flight platform, as in [CAS 05]. This configuration is shown in Figure ??. Therefore, using the equations (??) and (??), the rotational dynamics for the roll angle can be represented by:

$$
\ddot{\phi}=\ell / J_{x}
$$

where, the sum of moments $\ell$ can be calculated as follows:

$$
\ell=F \cdot d-C_{\ell_{\dot{\phi}}} \dot{\phi}
$$

and $F=f_{1}-f_{2}$ is the force difference between the right and left rotor and $d$ is the distance from the center of mass to each rotor. The second term in the right side of equation (1.37) represents an aerodynamic moment produced by the change of the roll rate, normally opposing to the roll moment, that is why, the derivative, $C_{\ell_{\dot{\phi}}}=0.36$, is known as roll damping derivative. Then equation (1.36) can be rewritten as follows:

$$
\ddot{\phi}=\left(F \cdot d-C_{\ell_{\dot{\phi}}} \dot{\phi}\right) / J_{x}
$$

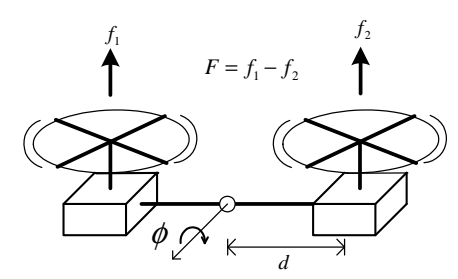

Figure 1.3: Approach of PVTOL to control the roll position

The lift force in each rotor can be considered as the thrust and can be calculated by the following expression:

$$
\mathcal{T}=C_{t} \rho n^{2} D_{p}^{4}
$$

where $C_{t}$ is the thrust coefficient, $\rho$ is the density of the air, $n$ is the number of revolutions per second of the motor and $D_{p}$ is the diameter of the propellers. The thrust 
coefficient is a function of the pitch angle propeller $\varphi$, which is shown in Figure ??. The thrust coefficient in a linear region can be calculated by:

$$
C_{t}=C_{t_{\varphi}} \varphi
$$

where $C_{t_{\varphi}}$ is a derivative which represents the thrust slope with respect to the VPP angle. This derivative has been estimated using a shareware program called JavaProp [HEP 06]. This program uses the number of blades, the velocity of rotation, the diameter of the propellers, the velocity and the power of the motor to give the value of $C_{t}$ for an operational range $5^{\circ} \leq \varphi \leq 15^{\circ}$ as is shown in Figure ??. Then, using Matlab $^{T M}$ a first order polynomial (dashed line) can be constructed using the values of the thrust coefficient for each $\varphi$ angle. The dashed line slope is the derivative of this polynomial which in fact represents the derivative $C_{t_{\varphi}}$, and its value is estimated to be 0.0025 .

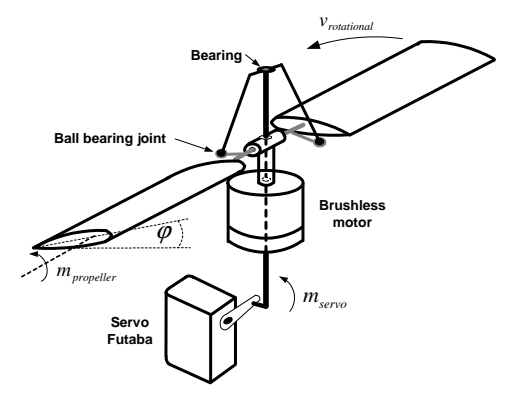

Figure 1.4: Schematic of Variable Pitch Propeller (VPP) System

\section{$C_{t}$ at different VPP angles}

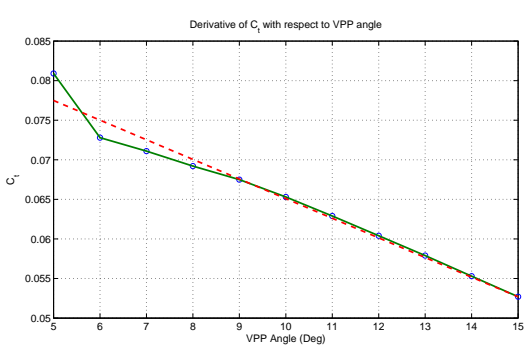

Then using the inertia values given in Table 1.1 and applying the Laplace transform, the following transfer function for the roll angle with respect to the VPP angle displacement is obtained.

$$
\frac{\phi(s)}{\varphi(s)}=\frac{5}{s^{2}+25 s}
$$


Now, the VPP dynamics will be determined. In the Figure ??, it can be seen that the aerodynamic pitch moment of the blades must be equal to the moment generated by the servo mechanism. Considering that the blade profile corresponds to the NACA0014, then the following approximation can be used to obtain the blade pitch moment:

$$
m_{b}=\frac{\rho V_{t_{b}}^{2} S_{b} \bar{c}_{b}}{2 J_{y_{b}}}\left[C_{m_{\varphi}} \varphi+C_{m_{\dot{\varphi}}} \dot{\varphi}\right]=k_{s} f_{s} \delta_{s}
$$

where the subscript $b$ denotes the blade. The term $V_{t_{b}}$ denotes the total velocity of the propeller at the tip, it is given by:

$$
V_{t_{b}}=\sqrt{v_{\text {axial }}^{2}+v_{\text {radial }}^{2}}
$$

where $v_{\text {radial }}=\pi n D$.

The term $C_{m_{\varphi}}=-0.0019$, is the estimated blade pitch moment coefficient slope with respect to $\varphi$, being obtained using Javafoil [HEP 06], an airfoil analysis shareware software. The term $C_{m_{\dot{\varphi}}}=1.6 \times 10^{-5}$ is a stability derivative generated by the variation of the VPP rate. The right hand side term of equation (1.42) represents the moment produced by the servo, where $f_{s}$ is the force produced by the servo, $\delta_{s}$ is the servo displacement and $k_{s}$ is a mechanical reduction factor. Using the parameter values in Table 1.1 and applying the Laplace transform, yields the VPP dynamic's transfer function:

$$
\frac{\varphi(s)}{\delta_{s}(s)}=\frac{120}{s+120}
$$

The actuator dynamics is given in [KAN 01] as follows:

$$
\frac{\delta_{s}(s)}{\delta_{c}(s)}=\frac{0.6}{0.1 s+1}
$$

Then using the transfer functions given previously, the control loop system shown in Figure ?? is proposed to stabilize the roll angle. The system is stable since the characteristic equation $0.1 s^{4}+15.5 s^{3}+445 s^{2}+4260 s+16200$ has all its roots in the left hand side of the complex plane, the roots are located at: $-6.7 \pm 4.5 i,-121$ and -20.5 .

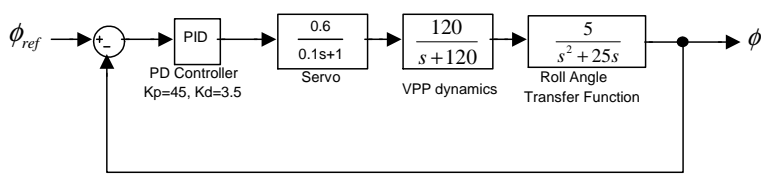

Figure 1.5: Roll Control Loop. 


\subsubsection{Pitch control}

To obtain the pitch control system the vehicle is considered to be a tailless aircraft flying in forward flight. Assuming that the roll angle is small enough and the roll rate is instantaneously zero, then using (??) and (??), a second order differential equation describing the rotational dynamics for the platform pitch angle can be written as:

$$
\ddot{\theta}=m / J_{y}
$$

where $m$ is the pitch moment of the wing, which is given by the following expression:

$$
m=\frac{1}{2} V^{2} \rho S \bar{c} C_{m}
$$

where $\bar{c}$ is the wing chord, $S$ is the wing reference area, $V$ is the airflow speed and $C_{m}$ is the pitching moment coefficient given by [ETK 96]:

$$
C_{m}=C_{m_{a c}}+C_{m_{\alpha}} \alpha+C_{m_{\delta_{e}}} \delta_{e}+C_{m_{q}} Q \frac{\bar{c}}{V}
$$

Assuming that in steady hover flight $\theta=\alpha$ and $C_{m_{a c}}=0$, then (1.46) can be reduced to:

$$
\ddot{\theta}=\frac{\rho V^{2} S \bar{c}}{2 J_{y}}\left[C_{m_{\alpha}} \theta+C_{m_{\delta_{e}}} \delta_{e}+C_{m_{q}} \frac{\bar{c}}{V} \dot{\theta}\right]
$$

The derivative $C_{m_{\alpha}}$ represents the variation of the pitching moment with respect to the angle-of-attack $\alpha$. This coefficient depends strongly on the airfoil profile. The derivative $C_{m_{\delta_{e}}}=\partial C_{m} / \partial q$ represents the variation of the pitching moment with respect to the elevator control. To estimate these parameters, a shareware software named JavaFoil has been used. This program allows the user to analyze and design, in a rapid and interactive way, a profile over a range of angles of attack, [HEP 06]. The vehicle main wing has a profile NACA0018 and its pitch moment curves at different angles of attack and elevator positions obtained with this program, are shown in Figure ??. Then $C_{m_{\alpha}}=-0.145$ and $C_{m_{\delta_{e}}}=0.65$. The derivative, $C_{m_{q}}=-10$, represents the aerodynamic effects due to rotations of the vehicle while the angle of attack remains zero. Using the vehicle parameters given in Table 1.1 and the Laplace transform, a second order transfer function representing the pitch angle dynamics is given as follows:

$$
\frac{\theta(s)}{\delta_{e}(s)}=\frac{85}{s^{2}+40 s+18}
$$

Then, using the actuator dynamics given previously, a simple proportional derivative compensator with $K_{p}=80$ and $K_{d}=17$, is proposed to stabilize the pitch angle. This controller stabilizes the platform pitch angle system because the roots of the characteristic equation $0.1 s^{3}+5 s^{2}+908.8 s+4098$ are located at $-22.7 \pm 91.45 i$ and -4.62 which are in the left hand side of the complex plane. 


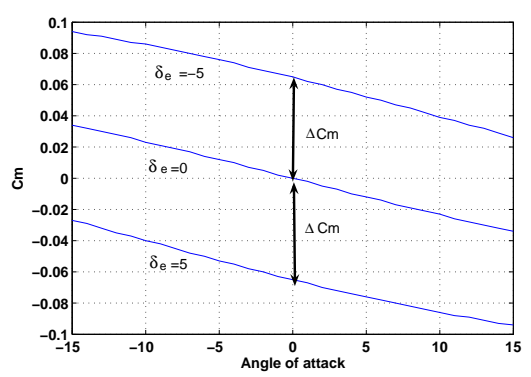

Figure 1.6: Pitch moment coefficient curves

\subsubsection{Yaw control}

Now, to control the vehicle yaw position, it is assumed that the pitch and roll angles are stabilized, then the roll rate and pitch rate vanish, then equation (??) can be written as follows:

$$
\ddot{\psi}=n / J_{z}
$$

where $n$ is the vehicle yaw moment. Notice that $n$ is used to control yaw during hover flight and to control roll during forward flight as shown in Figure ??. Under this assumption, the yaw moment can be approximated by the following expression

$$
n=\rho V^{2} S b C_{n} / 4
$$

where $b$ is the wing span and $C_{n}$ is the yawing moment coefficient given by $C_{n}=$ $C_{n_{\dot{\psi}}} \dot{\psi}+C_{n_{\delta_{e}}} \delta_{e}$. Then, (1.51) can be rewritten as

$$
\ddot{\psi}=\left(\rho V^{2} S b\right)\left(C_{n_{\dot{\psi}}} \dot{\psi}+C_{n_{\delta_{e}}} \delta_{e}\right) / 4 J_{z}
$$

where $C_{n_{\delta_{e}}}=0.19$ represents the variation of the yaw moment with respect to the ailerons positions. $C_{n_{\dot{\psi}}}=0.19$ is the yaw damping derivative. Applying Laplace transform and using numerical values yields:

$$
\frac{\psi(s)}{\delta_{e}(s)}=\frac{20}{s^{2}+20 s}
$$

Then, using the actuator dynamics given before, a closed-loop control system with a proportional derivative controller can be proposed, where $K_{p}=68$ and $K_{d}=17$. The characteristic equation is $0.1 s^{3}+3 s^{2}+224 s+20$ and its roots are located at $-13 \pm 44.4 i$ and -3.8 , therefore the system is stable.

\subsubsection{Simulation Results}

We have developed several simulations of the model to determine its qualities of flight. The following graphs shows stable dynamics using a PD controller. The 


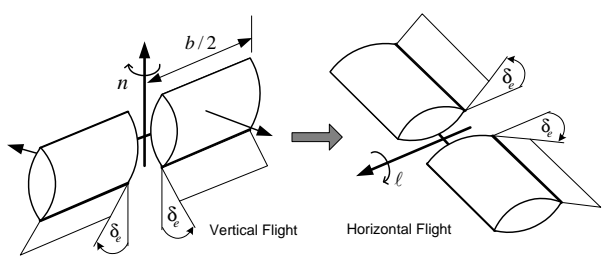

Figure 1.7:

Yaw Con-

trol.

Table 1.1: Bidule-CSyRex Aircraft Parameters

\begin{tabular}{lll}
\hline Parameter Value & Definition \\
\hline$S$ & $0.18 \mathrm{~m}^{2}$ & Wing Reference area \\
$\bar{c}$ & $0.3 \mathrm{~m}$ & Wing chord \\
$b$ & $0.6 \mathrm{~m}$ & Wing span \\
$\rho$ & $1.225 \mathrm{~kg} / \mathrm{m}^{3}$ & Air density \\
$V$ & $10 \mathrm{~m} / \mathrm{s}$ & Wind velocity (airflow) \\
$J_{x}$ & $0.0144 \mathrm{~kg} . \mathrm{m}$ & x-axis moment of inertia \\
$J_{y}$ & $0.0254 \mathrm{~kg} . \mathrm{m}$ & y-axis moment of inertia \\
$J_{z}$ & $0.0312 \mathrm{~kg} \cdot \mathrm{m}$ & z-axis moment of inertia \\
$d$ & $0.2 \mathrm{~m}$ & Rotor distance from the center of mass \\
$D$ & 0.27 & Propeller diameter \\
$\mathrm{n}$ & $9000 \mathrm{RPM}$ & Rotor speed \\
$S_{b}$ & $0.006 \mathrm{~m}^{2}$ & Blade reference area \\
$\bar{c}_{b}$ & $0.3 \mathrm{~m}$ & Blade chord \\
$J_{y_{b}}$ & $2 \times 10^{-6}$ & y-axis blade inertia \\
$f_{s}$ & $17 N$ & Force produced by the servo \\
\hline
\end{tabular}

response of the roll subsystem to a unit step is shown in Figure ??. It is clear that VPP control can stabilize the system very fast while speed control can not stabilize this system at all.

For each control loop the step response is evaluated. First, the roll control system is validated in simulation, this system based in the VPP mechanism has been compared with a roll control system based on the speed variation of the rotors. Normally, a control based on the speed variation introduces a time-delay, which is caused by the electronic of the speed controller. This time-delay, provokes instability in the system making the tuning of the controller parameters a very difficult task. Figure ?? shows the comparison of the two systems. The two systems reach the desired value almost at the same time, but in the system using speed variation there are oscillations in the 
steady state, while the VPP control quickly stabilizes the system. In the same way, the step response for the pitch and yaw closed-loop control systems have been simulated.

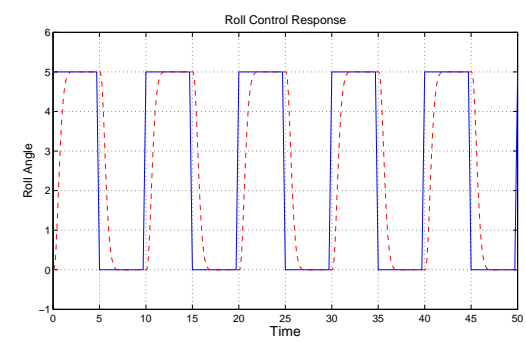

Figure 1.8: Response of the Roll Control Loop

\subsubsection{Experimental Results}

In this section, qualitative results in flight test of the tail-sitter are discussed. This vehicle in vertical flight presents a natural unstable behavior, and the manual guidance and control is a very difficult task even if the remote human operator has an excellent piloting skill. Figure ?? shows the vehicle crashing due to the high instability in a test without any automatic control algorithm.

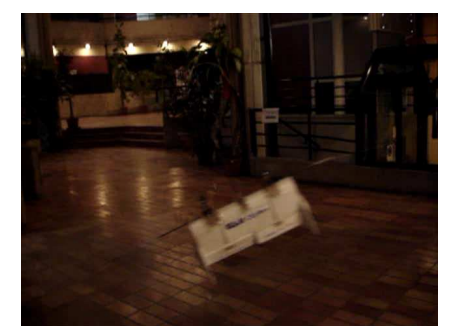

Figure 1.9: Bidule-CSyRex with no control

As it was seen in previous sections, the control law for this vehicle is a simple PD control, which has been chosen due that the position variables and its derivatives are obtained directly from the IMU. The integral is avoided due to the high probability for error in the steady state because of the signal noise in the sensors. To adjust the control parameters several flight tests were carried out until obtaining a good performance of the vehicle. First the $K_{d}$ gains were adjusted to get a good stiffness in all the angular displacements, then the $K_{p}$ gain was adjusted to obtain a good time response 
to changes of angular position. The stability derivatives, $C_{\ell_{\dot{\phi}}}, C_{m_{q}}, C_{n_{\delta_{e}}}, C_{n_{\dot{\psi}}}$ and $C_{m_{\dot{\varphi}}}$ would normally be estimated using the data obtained from wind tunnel tests. However, in the current study first the controller parameters were first obtained in flight test, then using the values of the derivatives and the aerodynamic coefficients estimated using Javafoil and Javaprop, the unknown derivatives were obtained. Figure ?? shows the vehicle flying stable when the linear control PD is used. Note that tethers were used for safety purposes only, with satisfactory flight test results used only when all the tether are slack, thus not supporting the flight platform in any way.

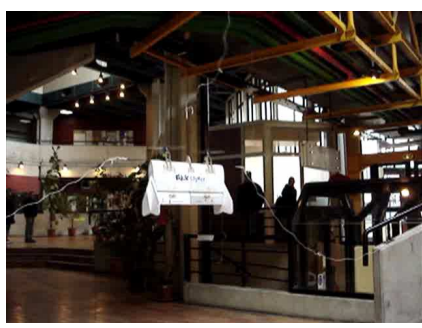

Figure 1.10: BiduleCSyRex with PD control

\subsubsection{Robust Control Considering Parametric Uncertainty}

It is usual to handle inaccurate mathematical models. In the previous section, aerodynamic coefficients for a tail-sitter had been estimated using shareware software. Then, mathematical model obtained for the tail-sitter vehicle may not be accurate due to estimation errors and due to imperfections in the vehicle building process. Thus, in this section a robust control design and analysis for a tail-sitter is developed.

Let us recall the roll subsystem

$$
\begin{aligned}
\ddot{\phi} & =\left(C_{t_{\varphi}} \varphi \rho n^{2} D^{4} \cdot d-C_{\ell_{\dot{\phi}}} \dot{\phi}\right) / J_{x} \\
{\left[C_{m_{\varphi}} \varphi+C_{m_{\dot{\varphi}}} \dot{\varphi}\right] } & =k_{s} f_{s} \delta_{s} \frac{2 J_{y_{b}}}{\rho V_{t_{b}}^{2} S_{b} \bar{c}_{b}}
\end{aligned}
$$

Then, the roll subsystem can be expressed as follows

$$
\begin{aligned}
\dot{\boldsymbol{\eta}}_{r} & =\boldsymbol{A} \boldsymbol{\eta}_{\boldsymbol{r}}+\boldsymbol{B} \delta_{c} \\
y & =\boldsymbol{C} \boldsymbol{\eta}_{\boldsymbol{r}}
\end{aligned}
$$


where

$$
\boldsymbol{A}=\left[\begin{array}{cccc}
0 & 1 & 0 & 0 \\
0 & 0 & 1 & 0 \\
0 & 0 & 0 & 1 \\
0 & -30000 & -4450 & -155
\end{array}\right], \boldsymbol{B}=\left[\begin{array}{c}
0 \\
0 \\
0 \\
3600
\end{array}\right]
$$

and

$$
\boldsymbol{\eta}=\left[\begin{array}{c}
\phi \\
\dot{\phi} \\
-25 \phi+5 \varphi \\
625 \dot{\phi}-725 \varphi+600 \delta_{s}
\end{array}\right], \boldsymbol{C}^{T}=\left[\begin{array}{l}
1 \\
0 \\
0 \\
0
\end{array}\right]
$$

These values were obtained using the parameters values in Table 1.1. It is important to note that some of the aircraft parameters were obtained by experimental tests and the remaining aircraft parameters were estimated using shareware software. In order to take into account possible measurement errors, we will consider uncertainty in the last row coefficients of the state matrix. In spite of the uncertainty structure in the coefficients of the last row of the matrix, it is always possible to lump the uncertainty such that the resulting polynomial family is a lumped version of the original interval polynomial family. As a result of this considerations, the following matrix is obtained

$$
\boldsymbol{A}(\boldsymbol{q})=\left[\begin{array}{cccc}
0 & 1 & 0 & 0 \\
0 & 0 & 1 & 0 \\
0 & 0 & 0 & 1 \\
q_{0} & q_{1} & q_{2} & q_{3}
\end{array}\right]
$$

where $q_{0} \in[-10,10], q_{1} \in[-36000,-24000], q_{2} \in[-5340,-3560]$ and $q_{3} \in$ $[-186,-124]$.

\subsubsection{Pitch Subsystem}

The pitch angle dynamics (1.48) can also be written as

$$
\begin{aligned}
\dot{\boldsymbol{\eta}}_{p} & =\boldsymbol{A} \boldsymbol{\xi}_{\boldsymbol{p}}+\boldsymbol{B} \delta_{e} \\
y & =\boldsymbol{C} \boldsymbol{\xi}_{p}
\end{aligned}
$$

Using the vehicle parameters given in Table 1.1 and the same procedure for the roll subsystem leads to

$$
\boldsymbol{A}(\boldsymbol{q})=\left[\begin{array}{cc}
0 & 1 \\
-q_{0} & -q_{1}
\end{array}\right]
$$

with $q_{0}=[-21.6,-14.4], q_{1}=[-48,-32]$. 


\subsubsection{Yaw Subsystem}

The yaw angle dynamics (1.53) can also be written as

$$
\begin{aligned}
\dot{\boldsymbol{\eta}}_{y} & =\boldsymbol{A} \boldsymbol{\eta}_{\boldsymbol{y}}+\boldsymbol{B} \delta_{e} \\
y & =\boldsymbol{C} \boldsymbol{\eta}_{\boldsymbol{y}}
\end{aligned}
$$

Using the vehicle parameters given in table 1.1 and the same procedure used in roll subsystem we obtain

$$
\boldsymbol{A}(\boldsymbol{q})=\left[\begin{array}{cc}
0 & 1 \\
-q_{0} & -q_{1}
\end{array}\right]
$$

with $q_{0}=0, q_{1}=[-24,-16]$.

Now on our goal is to present a robust state feedback control design to stabilize a system with uncertain parameter values. Subsequently, the value set characterization is used to verify the robust stability property when a time delay in the process is considered. To do this, the Bidule CSyRex roll subsystem (1.57) and (1.59) will be represented in the form:

$$
\Sigma_{u n} \triangleq\left\{\begin{array}{c}
\dot{\boldsymbol{\eta}}=\boldsymbol{A}\left(\boldsymbol{q}^{-}\right) \boldsymbol{\eta}+\boldsymbol{B} u+\boldsymbol{B} \boldsymbol{\Gamma}(r) \boldsymbol{\eta} \\
y=\boldsymbol{C} \boldsymbol{\eta}
\end{array}\right.
$$

where

$$
\begin{aligned}
\boldsymbol{A}\left(\mathbf{q}^{-}\right) & =\left[\begin{array}{cccc}
0 & 1 & 0 & 0 \\
0 & 0 & 1 & 0 \\
0 & 0 & 0 & 1 \\
-10 & -36000 & -5340 & -186
\end{array}\right] \\
\boldsymbol{\Gamma}(r) & =\left[\begin{array}{llll}
r_{3} & r_{2} & r_{1} & r_{0}
\end{array}\right]
\end{aligned}
$$

where $r_{0} \in[0,0.0172] ; r_{1} \in[0,0.4944] ; r_{2} \in[0,3.3333] ; r_{3} \in[0,0.0056]$. Now, the $\boldsymbol{F}$ matrix is defined in such a way that the following condition is satisfied:

$$
\boldsymbol{\Gamma}(r)^{T} \boldsymbol{\Gamma}(r) \leq \boldsymbol{F} \quad \forall r \in \mathbb{R}
$$


and the nominal system will be considered as follows:

$$
\Sigma_{n o m} \triangleq\left\{\begin{array}{c}
\dot{\boldsymbol{\eta}}=\boldsymbol{A}\left(\boldsymbol{q}^{-}\right) \boldsymbol{\eta}+\boldsymbol{B} u \\
y=\boldsymbol{C} \boldsymbol{\eta}
\end{array}\right.
$$

With the above definitions, it is possible to present the following result:

PROPOSITION 1.1.- Consider the Bidule CSyRex roll subsystem (1.66), and the following control law

$$
u=-\boldsymbol{B}^{T} \boldsymbol{S} \boldsymbol{\eta}
$$

where

$$
\boldsymbol{S A}\left(\boldsymbol{q}^{-}\right)+\boldsymbol{A}\left(\boldsymbol{q}^{-}\right)^{T} \boldsymbol{S}+\boldsymbol{F}+\boldsymbol{I}-\boldsymbol{S} \boldsymbol{B} \boldsymbol{B}^{T} \boldsymbol{S}=0, \boldsymbol{S}>0
$$

for system (1.66), the control law is:

$$
u=-10 \eta_{1}-1.5428 \eta_{2}-0.1173 \eta_{3}-0.0034 \eta_{4}
$$

then, the closed-loop system is robustly stable.

Proof. considering the following Lyapunov candidate function:

$$
V(\boldsymbol{\eta})=\min _{u \in R} \int_{0}^{\infty}\left(\boldsymbol{\eta}^{T} \boldsymbol{F} \boldsymbol{\eta}+\boldsymbol{\eta}^{T} \boldsymbol{\eta}+u^{T} u\right) d t
$$

It is possible to verify that the proposed control law (1.71) corresponds to the solution of the LQR optimal control problem for the $\Sigma_{n o m}$ system (1.68), considering the cost functional $V(\boldsymbol{\eta})$, and the relative weights matrices $\boldsymbol{H}=\boldsymbol{F}+\boldsymbol{I}$ and $\mathbf{R}=1$. Obviously, the above control law stabilizes the nominal system $\Sigma_{n o m}$. Next, a proof that the same control law also stabilizes the uncertain system $\Sigma_{u n}$ will be presented. Using the results of the LQR optimal control problem, it is possible to obtain the following solution to the problem in (1.72):

$$
V^{*}(\boldsymbol{\eta})=\int_{0}^{\infty}\left(\boldsymbol{\eta}^{T} \boldsymbol{F} \boldsymbol{\eta}+\boldsymbol{\eta}^{T} \boldsymbol{\eta}+\boldsymbol{\eta}^{T} \boldsymbol{S} \boldsymbol{B} \boldsymbol{B}^{T} \boldsymbol{S} \boldsymbol{\eta}\right) d t
$$

by definition, $V^{*}(\boldsymbol{\eta})$ must satisfy the Hamilton-Jacobi-Bellman equations, see [AND 90], [LEW 95] and [LIN 07]:

$$
\begin{gathered}
\boldsymbol{\eta}^{T} \boldsymbol{F} \boldsymbol{\eta}+\boldsymbol{\eta}^{T} \boldsymbol{\eta}+\boldsymbol{\eta}^{T} \boldsymbol{S} \boldsymbol{B} \boldsymbol{B}^{T} \boldsymbol{S} \boldsymbol{\eta} \\
+\left[\frac{\partial V(\boldsymbol{\eta})}{\partial \boldsymbol{\eta}}\right]^{T}\left(\boldsymbol{A}\left(\boldsymbol{q}^{-}\right) \boldsymbol{\eta}+\boldsymbol{B} \boldsymbol{B}^{T} \boldsymbol{S} \boldsymbol{\eta}\right)=0 \\
2 \boldsymbol{\eta}^{T} \boldsymbol{S} \boldsymbol{B}+\left[\frac{\partial V(\boldsymbol{\eta})}{\partial \boldsymbol{\eta}}\right]^{T} \boldsymbol{B}=0
\end{gathered}
$$


Along the trajectories of the system $\Sigma_{u n}(1.66)$ we have

$$
\begin{aligned}
\dot{V}(\boldsymbol{\eta})= & {\left[\frac{\partial V(\boldsymbol{\eta})}{\partial \boldsymbol{\eta}}\right]^{T} \dot{\boldsymbol{\eta}} } \\
= & {\left[\frac{\partial V(\boldsymbol{\eta})}{\partial \boldsymbol{\eta}}\right]^{T}\left[\boldsymbol{A}\left(\boldsymbol{q}^{-}\right) \boldsymbol{\eta}+\boldsymbol{B} \boldsymbol{u}+\boldsymbol{B} \boldsymbol{\Gamma}(r) \boldsymbol{\eta}\right] } \\
= & {\left[\frac{\partial V(\boldsymbol{\eta})}{\partial \boldsymbol{\eta}}\right]^{T}\left[\boldsymbol{A}\left(\boldsymbol{q}^{-}\right) \boldsymbol{\eta}+\boldsymbol{B} \boldsymbol{B}^{T} \boldsymbol{S} \boldsymbol{\eta}\right] } \\
& +\left[\frac{\partial V(\boldsymbol{\eta})}{\partial \boldsymbol{\eta}}\right]^{T} \boldsymbol{B} \boldsymbol{\Gamma}(r) \boldsymbol{\eta}
\end{aligned}
$$

Then (1.73) and (1.74), leads to

$$
\begin{aligned}
\dot{V}(\boldsymbol{\eta})= & -\boldsymbol{\eta}^{T} \boldsymbol{F} \boldsymbol{\eta}-\boldsymbol{\eta}^{T} \boldsymbol{\eta}-\boldsymbol{\eta}^{T} \boldsymbol{S} \boldsymbol{B} \boldsymbol{B}^{T} \boldsymbol{S} \boldsymbol{\eta} \\
& -2 \boldsymbol{\eta}^{T} \boldsymbol{S} \boldsymbol{B} \boldsymbol{\Gamma}(r) \boldsymbol{\eta} \\
= & -\boldsymbol{\eta}^{T} \boldsymbol{F} \boldsymbol{\eta}-\boldsymbol{\eta}^{T} \boldsymbol{\eta}-\boldsymbol{\eta}^{T} \boldsymbol{S} \boldsymbol{B} \boldsymbol{B}^{T} \boldsymbol{S} \boldsymbol{\eta} \\
& -2 \boldsymbol{\eta}^{T} \boldsymbol{S} \boldsymbol{B} \boldsymbol{\Gamma}(r) \boldsymbol{\eta} \pm \boldsymbol{\eta}^{T} \boldsymbol{\Gamma}^{T}(r) \boldsymbol{\Gamma}(r) \boldsymbol{\eta} \\
= & -\boldsymbol{\eta}^{T}\left[\boldsymbol{F}-\boldsymbol{\Gamma}^{T}(r) \boldsymbol{\Gamma}(r)\right] \boldsymbol{\eta}-\boldsymbol{\eta}^{T} \boldsymbol{\eta} \\
& -\boldsymbol{\eta}^{T}\left[\boldsymbol{B}^{T} \boldsymbol{S}+\boldsymbol{\Gamma}(r)\right]^{T}\left[\boldsymbol{B}^{T} \boldsymbol{S}+\boldsymbol{\Gamma}(r)\right] \boldsymbol{\eta}
\end{aligned}
$$

from condition (1.67) it follows

$$
\dot{V}(\boldsymbol{\eta}) \leq-\boldsymbol{\eta}^{T} \boldsymbol{\eta}
$$

Then, $\dot{V}(\boldsymbol{\eta})<0$ for all $\boldsymbol{\eta} \neq 0$ and $\dot{V}(\boldsymbol{\eta})=0$ if and only if $\boldsymbol{\eta}=0$, which ends the proof.

All processes have time delays due to sensor information process, actuator time delay, etc. Considering a time delay $\tau$, system (1.66) can be rewritten as

$$
\dot{\boldsymbol{\eta}}(t)=\left[\begin{array}{cccc}
0 & 1 & \ldots & 0 \\
0 & 0 & \ldots & 0 \\
\vdots & \vdots & \ddots & 1 \\
-q_{0} & -q_{1} & \ldots & -q_{n-1}
\end{array}\right] \boldsymbol{\eta}(t)+\left[\begin{array}{c}
0 \\
0 \\
0 \\
r_{0}
\end{array}\right] u(t-\tau)
$$


PROPOSITION 1.2.- Consider the Bidule CSyRex roll subsystem with time delay (1.76), then this system is robustly stable if the same control law (1.71) is used and the maximum time-delay is $\tau_{\max }=1$ sec.

Proof. The uncertain time-delay system (1.76) has the following characteristic equation:

$$
\begin{gathered}
p\left(s, \mathbf{q}, \mathbf{r}, e^{-\tau s}\right)=s^{4}+[124,186] s^{3} \\
+[3560,5340] s^{2}+[24000,36000] s+[-10,10] \\
+\left(12 s^{3}+422 s^{2}\right. \\
+5553 s+35990) e^{-[0,1] s}
\end{gathered}
$$

These kind of functions are known as quasipolynomials. It is clear that the above characteristic equation (1.77) represents an infinite number of quasipolynomials that have to be considered to verify the robust stability property. This family is defined as follows:

$$
\mathcal{P}_{\tau} \triangleq\left\{\begin{array}{c}
p\left(s, \mathbf{q}, \mathbf{r}, e^{-\tau s}\right): \mathbf{q} \in \mathcal{Q} \\
\mathbf{r} \in \mathcal{R} ; \tau \in\left[0, \tau_{\max }\right]
\end{array}\right\}
$$

where $\mathcal{Q}$ and $\mathcal{R}$ represent the set of uncertainty, see [BAR 94].

It is clear that the value set of $P_{\tau}$ is a set of complex numbers plotted on the complex plane for values of $\mathbf{q}, \mathbf{r}, \omega$ and $\tau$ inside the defined boundaries. Next, the zero exclusion principle is presented in order to verify the robust condition [BAR 94].

LEMma 1.1.- Consider the characteristic equation (1.77), also called quasipolynomials. Suppose that (1.77) has at least one stable member. Then the robust stability property of the control system is guaranteed if and only if:

$$
0 \notin V_{\tau}(\omega) \quad \forall \omega \geq 0
$$

The results presented in [ROM 95] and [ROM 97] permit building the value set $V_{\tau}(\omega)$ for the characteristic equation (1.77) and is presented in Figures ?? and ??.

It can be noted that the zero is not included in the value set $V_{\tau}(\omega)$. Then the system (1.76) is then robustly stable.

\subsubsection{Simulation}

To investigate the behavior of the control stabilization system, several simulations of the model have been run using Matlab Simulink ${ }^{T M}$. This helps determine the flight handling qualities of the vehicle. Roll control system is simulated using control law 


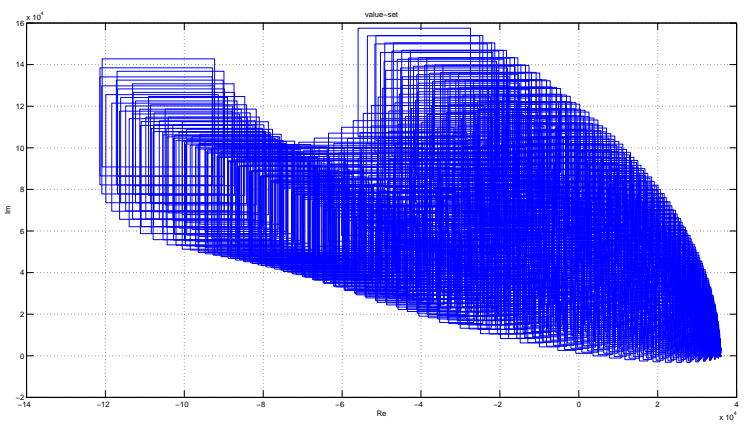

Figure 1.11: Value set of Roll subsystem control

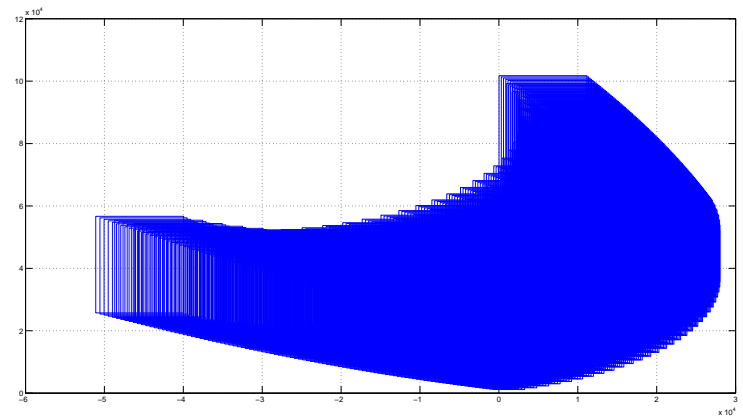

Figure 1.12:

Value set

zoom

(1.71). Its behavior is shown in Figure ??. In the same way, the pitch and yaw closedloop control systems have been simulated. Their respective responses are shown in Figures ?? and ??.

\subsubsection{Nonlinear Control based on Nested Saturations}

Linear and robust control approaches to attitude stabilization of a mini tail-sitter have been introduced in sections 1.3.1 and 1.3.2. Now, a nonlinear control strategy based on nested saturations to stabilize attitude and position of a mini tail-sitter 


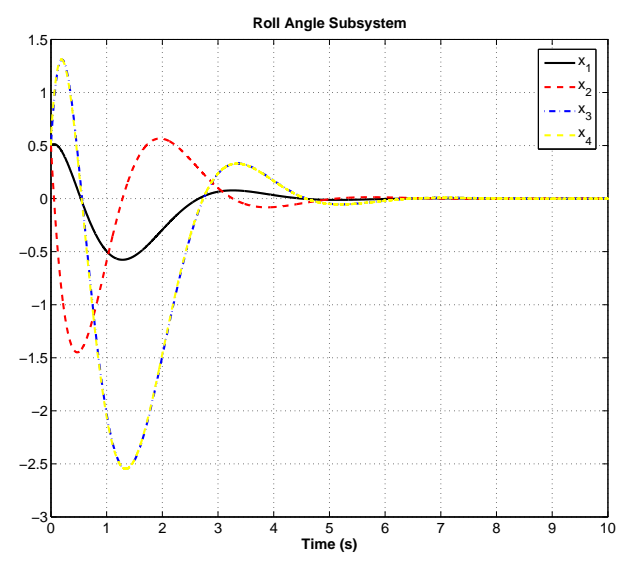

Figure 1.13: Response of the Roll control loop

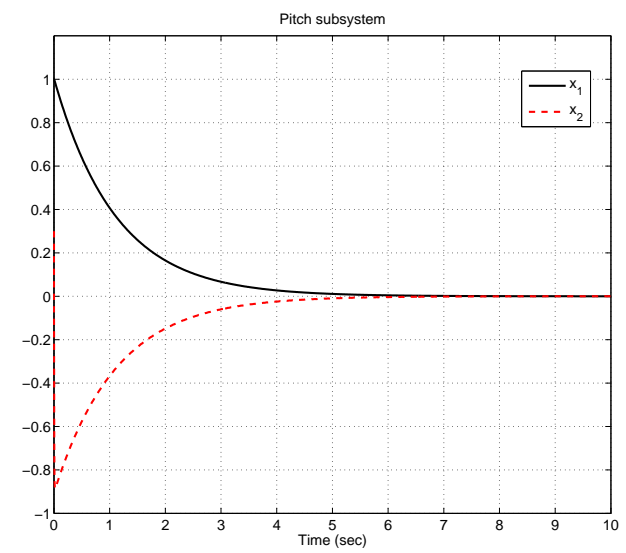

Figure 1.14: Response of the Pitch control loop

shown in Figure ?? in hover flight is introduced. Let us recall the attitude dynamic equations for mini UAVs ?? and ?? where $\boldsymbol{M}_{\boldsymbol{A}, \boldsymbol{T}}^{\boldsymbol{b}}$ represents the aerodynamic and thrust moments. Then, applying a control strategy similar to the one presented for the quadrotor vehicle, the following control input is proposed

$$
\boldsymbol{M}_{A / T}^{b} \triangleq \Omega_{b / e}^{b} \boldsymbol{J}^{b} \omega_{b / e}^{b}+\boldsymbol{H}(\Phi)^{-1} J^{b}\left[\tilde{\tau}-\dot{H}(\Phi) \omega_{b / e}^{b}\right]
$$




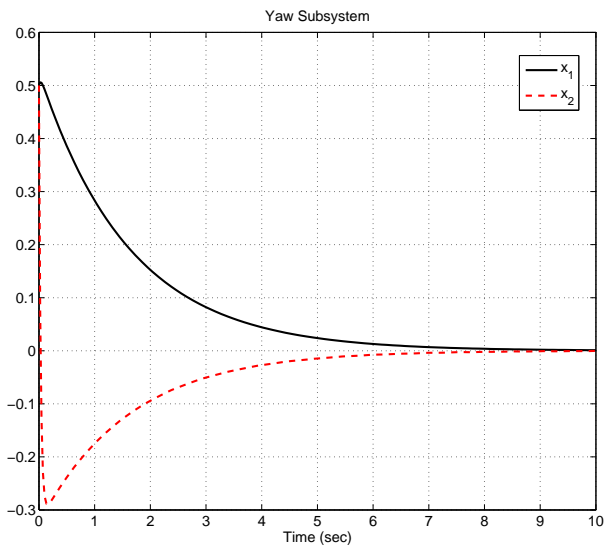

Figure 1.15: Response of the Yaw control loop

where $\tilde{\boldsymbol{\tau}}=\left[\begin{array}{ccc}\tilde{\tau_{\phi}} & \tilde{\tau_{\theta}} & \tilde{\tau_{\psi}}\end{array}\right]^{T}$. Then (??) can be rewritten as

$$
\begin{aligned}
& \ddot{\phi}=\tilde{\tau}_{\phi} \\
& \ddot{\theta}=\tilde{\tau}_{\theta} \\
& \ddot{\psi}=\tilde{\tau}_{\psi}
\end{aligned}
$$

\subsubsection{Equations of Translational Motion}

The translational motion equations can be obtained using equations (??) and (??). Then the kinematic equations of translational motion are written as

$$
\begin{aligned}
\dot{U} & =f_{x} / m-g s \theta+R V-Q W \\
\dot{V} & =f_{y} / m+g s \phi c \theta+R U-P W \\
\dot{W} & =f_{z} / m+g c \phi c \theta+Q U-P V
\end{aligned}
$$

Since the vehicle is a basic airfoil profile NACA0018, the aerodynamic force has two components, lift over $x$-axis and drag over $z$-axis, the vehicle forces are given by 
$f_{x}=L_{e_{1}}+L_{e_{2}}, f_{y}=0$ and $f_{z}=F_{1}+F_{2}-g-D_{e_{1}}+D_{e_{2}}$ where $L_{e_{1}}$ and $L_{e_{2}}$ are the lift forces in each elevon, $D_{e_{1}}$ and $D_{e_{2}}$ are the drag forces due to the elevons and $F_{1}$ and $F_{2}$ are the forces due to thrust of the motors. These forces are given as

$$
\begin{aligned}
L_{e_{i}} & =\frac{1}{2} \rho V^{2} S C_{L} \\
D_{e_{i}} & =\frac{1}{2} \rho V^{2} S C_{D}
\end{aligned}
$$

where $C_{L}$ and $C_{D}$ are the lift and drag coefficients which can be calculated using shareware programs as Javafoil.

Assuming that the translational and angular motion are decoupled, i.e. the euler angles and angular rates are zero, then the translational motion equations can be rewritten as

$$
\left[\begin{array}{c}
\ddot{x} \\
\ddot{y} \\
\ddot{z}
\end{array}\right]=\left[\begin{array}{ccc}
c \theta c \psi & s \phi s \psi+c \phi s \theta c \psi & 0 \\
c \theta s \psi & -s \phi c \psi+c \phi s \theta s \psi & 0 \\
-s \theta & c \phi c \theta & 1
\end{array}\right]\left[\begin{array}{c}
\frac{f_{x}}{m} \\
\frac{f_{z}}{m} \\
-g
\end{array}\right]
$$

In this case a four integrators in cascade to stabilize roll is presented. The same recursive algorithm is used to stabilize pitch.

Now, it is possible to introduce the following theorem :

THEOREM 1.1.- Consider the vehicle dynamics (1.89), (1.81)-(1.83) with control input $f_{x} \triangleq(\theta-g \sin \theta) / \cos \theta$ and $f_{z} \triangleq(r+g) / \cos \theta$ with $r \triangleq-k_{1} \dot{z}-k_{2}\left(z-z_{d}\right)$,

$$
\begin{aligned}
& \tilde{\tau}_{\theta}=-\sigma_{n}\left(\chi_{n}(x)+\sigma_{n-1}\left(\chi_{n-1}(x)+\ldots+\sigma_{1}\left(\chi_{1}(x)\right)\right)\right) \\
& \tilde{\tau}_{\phi}=-\sigma_{n}\left(\bar{\chi}_{n}(x)+\sigma_{n-1}\left(\bar{\chi}_{n-1}(x)+\ldots+\sigma_{1}\left(\bar{\chi}_{1}(x)\right)\right)\right)
\end{aligned}
$$

with for $n=1, \ldots, 4$ for both $\theta$ subsystem and $\phi$ subsystem and $\tilde{\tau}_{\psi}=-k_{3} \dot{\psi}-$ $k_{4}\left(\psi-\psi_{d}\right)$ for $\psi$ subsystem. The functions $\sigma_{i}$ are differentiable linear saturations. Let $\mathfrak{b} \equiv[x, \dot{x}, y, \dot{y}, z, \dot{z}, \phi, \dot{\phi}, \theta, \dot{\theta}, \psi, \dot{\psi}]$. Then for any $\mathfrak{b}(0) \in \mathbb{R}^{12}, \lim _{t \rightarrow \infty} \mathfrak{b}(t)=$ 0 .

Proof. The aim of the proposed nonlinear control is to stabilize the vehicle in hover flight. Then, we develop a control for longitudinal, lateral and axial dynamics. Thus, 
we start the proof by considering the translational dynamic equations (1.89), then the translational dynamics is reduced to

$$
\begin{aligned}
& \ddot{y}=-f_{z} \sin \phi \\
& \ddot{z}=f_{z} \cos \phi-g
\end{aligned}
$$

To stabilize the altitude, it is proposed that $f_{z}=(r+g) / \cos \phi$ where $r=-k_{1} \dot{z}-$ $k_{2}\left(z-z_{d}\right)$ with $k_{1}, k_{2}>0$ and $z_{d}$ is the desired altitude. Since $r \rightarrow 0$ it can be concluded that $z \rightarrow z_{d}$.

Then, equation (1.92) becomes $\ddot{y}=-g \tan \phi$. Assuming that the vehicle evolves in a neighborhood $\|\phi\|<\pi / 10$ Therefore, the lateral dynamics reduces to four integrators in cascade as follows

$$
\begin{aligned}
& \ddot{y}=-g \phi \\
& \ddot{\phi}=\tau_{\varphi}
\end{aligned}
$$

Assuming that the roll angle and yaw angle are stabilized, the translational dynamic equations (1.89) are reduced to

$$
\begin{aligned}
& \ddot{x}=c \theta f_{x} / m+s \theta f_{z} / m \\
& \ddot{z}=-s \theta f_{x} / m+c \theta f_{z} / m-g
\end{aligned}
$$

Assuming that for a time long enough to make $f_{z} \approx g$ and using the control input

$$
f x \triangleq \frac{\theta-g \sin \theta}{\cos \theta}
$$

Then, the longitudinal model is reduced to a four integrators in cascade

$$
\begin{aligned}
& \ddot{x}=\theta \\
& \ddot{\theta}=\tau_{\theta}
\end{aligned}
$$

Considering that the pitch and roll angles are stabilized, a simple PD controller can be proposed to stabilize the yaw attitude. Therefore, $\tau_{\psi}=-k_{3} \dot{\psi}-k_{4} \psi$ with $k_{3}, k_{4}>0$. This control is such that $\psi \rightarrow 0$. 
Now, introducing the following variables

$$
\begin{aligned}
& \chi_{1}=\chi_{2}+\dot{y}+2 \phi+\dot{\phi} \\
& \chi_{2}=\chi_{3}+\phi+\dot{\phi} \\
& \chi_{3}=\dot{\phi}+\phi \\
& \chi_{4}=\dot{\phi}
\end{aligned}
$$

To simplify the analysis, a recursive methodology is proposed. To do this, it is assumed that

$$
\begin{aligned}
\zeta_{n} & \left.=\chi_{n}(x)+\sigma_{n-1}\left(\zeta_{n-1}(x)\right)\right) \\
\zeta_{1} & =\chi_{1}(x)
\end{aligned}
$$

and

$$
\left.u=-\sigma_{n}\left(\zeta_{n}\right)\right)
$$

Let us define the following positive definite function

$$
V_{n}=(1 / 2) \chi_{n}^{2}
$$

Differentiating V with respect to time, we obtain

$$
\dot{V}_{n}=\chi_{n} \dot{\chi}_{n}
$$

from the fact that $\dot{\chi}_{n}=-\sigma_{n}\left(\zeta_{n}\right)$, we have

$$
\left.\dot{V}_{n}=\chi_{n} u=-\chi_{n} \sigma_{n}\left(\zeta_{n}\right)\right)
$$

due to equation (1.105) we get

$$
\left.\dot{V}_{n}=-\chi_{n} \sigma_{n}\left(\chi_{n}(x)+\sigma_{n-1}\left(\zeta_{n-1}(x)\right)\right)\right)
$$

Using definition (??) and the condition from theorem (1.1), that $M_{n-1}<0.5 L_{n}$, it can be noted that if $\left|\chi_{n}\right|>0.5 L_{n}$ then $\dot{V}_{n}<0$. This means that there exist a time $T_{n}$ such that $\left|\chi_{n}\right| \leq 0.5 L_{n}$ for $\forall t>T_{n}$ which implies that $\left|\chi_{n}+\sigma_{n-1}\left(\zeta_{n-1}(x)\right)\right| \leq$ $0.5 L_{n}+M_{n-1} \leq L_{n}$.

When $n=1$ we have the base case of the recursion. This case is treated a little different, let us propose

$$
V_{1}=(1 / 2) \chi_{1}^{2}
$$


Differentiating V with respect to time, we obtain

$$
\dot{V}_{1}=\chi_{1} \dot{\chi}_{1}
$$

using (1.101)-(1.104) is possible to see that $\dot{\chi}_{1}=-\sigma_{1}\left(\chi_{1}\right)$, then we have

$$
\left.\dot{V}_{1}=-\chi_{1} \sigma_{1}\left(\zeta_{1}\right)\right)
$$

due to equation (1.106) we get

$$
\dot{V}_{1}=-\chi_{1} \sigma_{1}\left(\chi_{1}\right)
$$

As in the recursive case, it can be noted that if $\left|\chi_{1}\right|>0.5 L_{1}$ then $\dot{V}_{1}<0$. This means that there exist a time $T_{1}$ such that $\left|\chi_{1}\right| \leq 0.5 L_{1}$ for $\forall t>T_{1}$. It is important to note that $T_{n}<T_{n-1}$ for all $n>2$.

Since $\dot{V}_{1}<0$ then, from equations (1.106) and (1.112) implies that $\chi_{1}=\zeta_{1} \rightarrow 0$. It can be noted that starting from $i=2$ until $i=n$ we have the following set of equations due to the recursion of the method

$$
\begin{aligned}
& \dot{V}_{2}=-\chi_{2} \sigma_{2}\left(\chi_{2}(x)+\sigma_{1}\left(\zeta_{1}(x)\right)\right) \\
& \dot{V}_{3}=-\chi_{3} \sigma_{3}\left(\chi_{3}(x)+\sigma_{2}\left(\zeta_{2}(x)\right)\right) \\
& \dot{V}_{4}=-\chi_{4} \sigma_{4}\left(\chi_{4}(x)+\sigma_{3}\left(\zeta_{3}(x)\right)\right)
\end{aligned}
$$

The recursion of equation (1.105) leads us to:

$$
\begin{aligned}
& \left.\zeta_{2}=\chi_{2}(x)+\sigma_{1}\left(\zeta_{1}(x)\right)\right) \\
& \left.\zeta_{3}=\chi_{3}(x)+\sigma_{2}\left(\zeta_{2}(x)\right)\right) \\
& \left.\zeta_{4}=\chi_{4}(x)+\sigma_{3}\left(\zeta_{3}(x)\right)\right)
\end{aligned}
$$

From (1.116), $\chi_{2} \rightarrow 0$, (1.119) implies that $\zeta_{2} \rightarrow 0$, in a recursive form (1.117), $\chi_{3} \rightarrow 0$, from (1.120) $\zeta_{3} \rightarrow 0$, from (1.118), $\chi_{4} \rightarrow 0$, from (1.121), $\zeta_{4} \rightarrow 0$. This means that, from (1.104) $\dot{\phi} \rightarrow 0$, from (1.103) $\phi \rightarrow 0$, from (1.102) $\dot{y} \rightarrow 0$, and finally from (1.101) $y \rightarrow 0$.

By using a set of variables similar to $\chi_{1}-\chi_{4}$ replacing $\phi$ by $\theta, y$-position by $x$ position and the same procedure described above for the $x-\theta$ subsystem, it is possible to show the stability of this subsystem. 


\subsubsection{Simulation Results}

To investigate the behavior of the control stabilization systems, several simulations of the model have been run using Matlab Simulink ${ }^{T M}$. Their respective responses are shown in Figures ?? and ??.

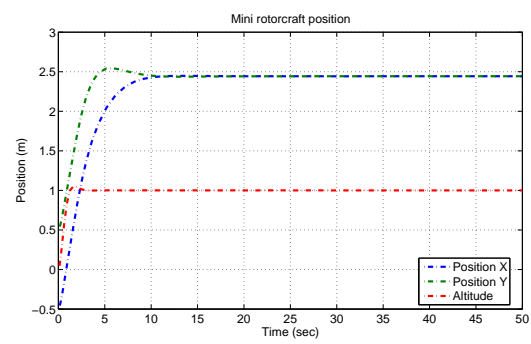

Figure 1.16: Response of the Roll Control Loop

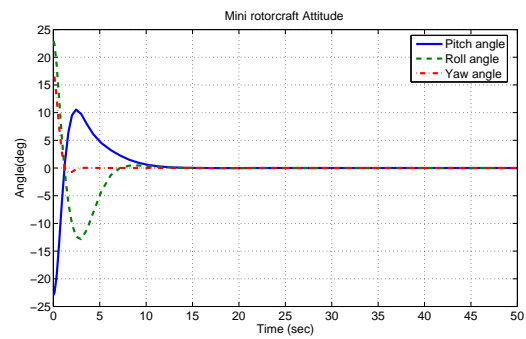

Figure 1.17: Response of the Pitch Control Loop

\subsection{Quad-Tilting Rotor Convertible MAV}

By marrying the take-off and landing capabilities of the helicopter with the forward flight efficiencies of fixed-wing aircraft, the Quad-plane promises a unique blend of capabilities at lower cost than other UAV configurations. While the tilt-rotor concept is very promising, it also comes with significant challenges. Indeed it is necessary to design controllers that will work over the complete flight envelope of the vehicle: from low-speed vertical flight through high-speed forward flight. The main change in this respect (besides understanding the detailed aerodynamics) is the large variation in the vehicle dynamics between these two different flight regimes. Several experimental platforms have been realized with a body structure in which the transition flight is executed by turning the complete body of the aircraft [GRE 06], [ESC 06], [STO 04], 


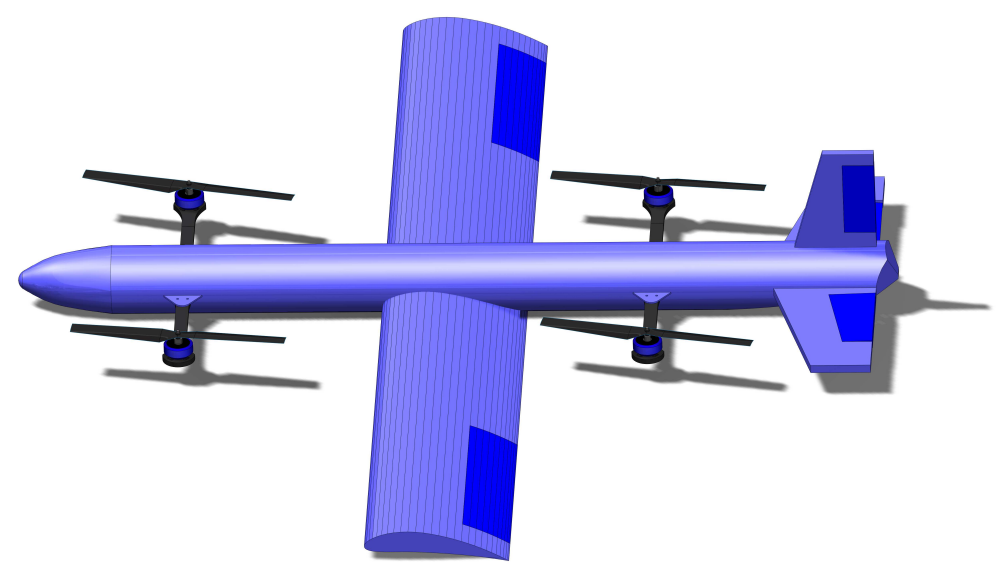

Figure 1.18: Quad-Tilting Rotor

Convertible UAV.

[STO 02b], [ESC 07]. In [STO 04] and [STO 02b] the authors described the development (modeling, control architecture and experimental prototype) of Two-rotor tailsitter. The control architecture features a complex switching logic of classical linear controllers to deal with the vertical, transition and forward flight. [GRE 06] presents a classical airplane configuration MAV to perform both operational modes. The hover flight is autonomously controlled by an onboard control flight system while the transition and cruise flight is manually controlled. A standard PD controller is employed during hover flight to command the rudder and elevator. In [ESC 06] some preliminary results are presented for the vertical flight of a Two-rotor MAV as well as a low-cost embedded flight control system. There are some examples to other tilt-rotor vehicles with quad-rotor configurations like Boeing's V44 [SNY 00] and the QTW UAV [NON 07]. In [ONE 08] the authors present the progress of their ongoing project, an aircraft with four tilting wings. A new tilt-rotor aircraft (Quad-plane Unmanned Aerial Vehicle) that is capable of flying in horizontal and vertical modes is presented in this section. The vehicle is driven by four rotors and has a conventional airplanelike structure, which constitutes a highly nonlinear plant and thus the control design should take into account this aspect. A nonlinear control strategy, consisting of a feedback-linearizable input for altitude control and a hierarchical control (inner-outer loop) scheme for the underactuated dynamic subsystem ( $x$-position, pitch), is proposed to stabilize the aerial robot within the hovering mode. Backstepping [KHA 02], a Lyapunov based method is presented to stabilize the vehicle within the airplane mode. Through the use recursive method, backstepping divides the control problem 


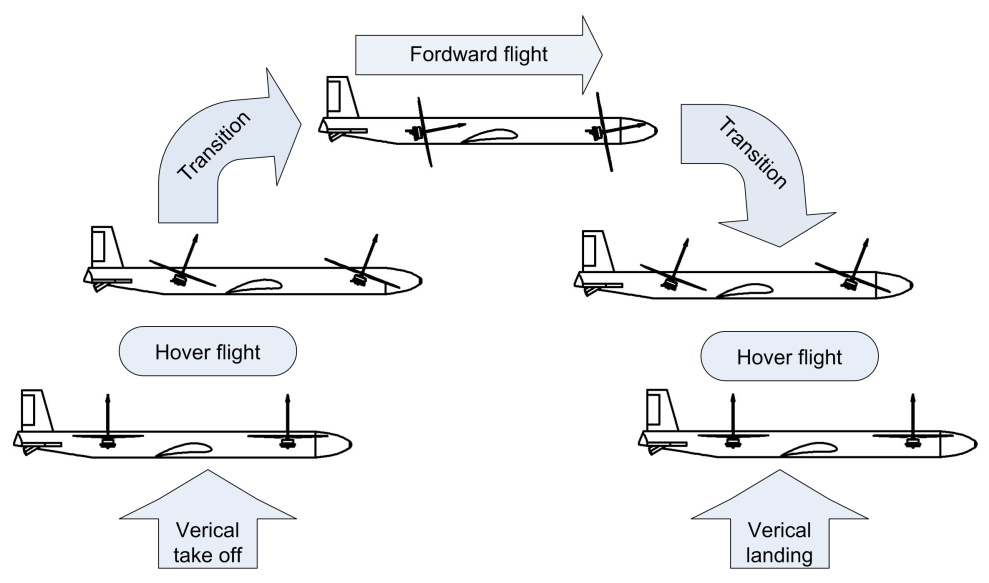

Figure 1.19: Opera-

tional transition.

into a sequence of designs for simpler systems. This mini aerial vehicle is one of the first of its kind among tilt-wing vehicles on that scale range.

\subsubsection{Modeling}

This section presents the longitudinal equations of motion as well as the aerodynamics of the vehicle. Due to the flight profile of the vehicle we distinguish three operation modes: (1) Hover Flight (HV) the aircraft behaves as a rotary-wing platform $\left(|\gamma| \leq \frac{\pi}{6}\right)$, (2) Slow-Forward Flight (SFF) $\left(\frac{\pi}{6}<|\gamma| \leq \frac{\pi}{3}\right)$ and finally (3) Fast-Forward Flight $(F F F)$, where the aerial robot behaves as a pure airborne vehicle $\left(\frac{\pi}{3}<|\gamma|<\frac{\pi}{2}\right)$.

1) During the $H F$ the 3D vehicle's motion relies only on the rotors. Within this phase the vehicle features VTOL flight profile. The controller for this regime disregard the aerodynamic terms due to the negligible translational speed.

2) It is possible to distinguish an intermediate operation mode, the SFF, which links the two flight conditions, $H F$ and $F F F$. This is probably the most complex dynamics.

3) FFF regime mode (Aft position), at this flight mode the aircraft has gained enough speed to generate aerodynamic forces to lift and control the vehicle motion.

\section{Kinematics}

$-\mathcal{F}^{i}$ denotes the inertial earth-fixed frame with origin, $O^{i}$, at the earth surface. This frame is associated to the vector basis $\left\{\boldsymbol{i}_{\boldsymbol{i}}, \boldsymbol{j}_{\boldsymbol{i}}, \boldsymbol{k}_{\boldsymbol{i}}\right\}$. 


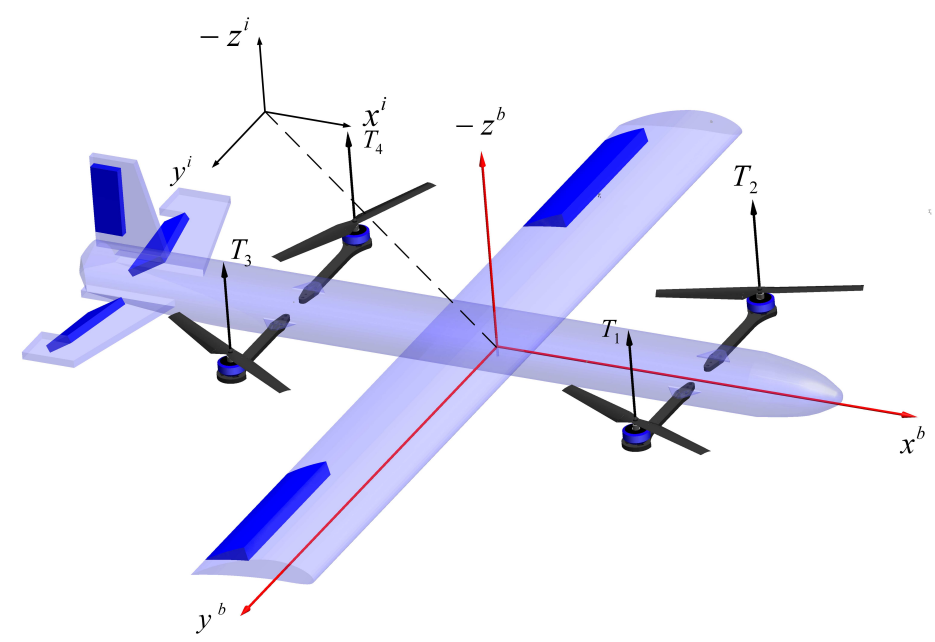

Figure 1.20: Coordinate systems: Inertial frame $\left(F^{i}\right)$ and Body-fixed frame $\left(F^{b}\right)$

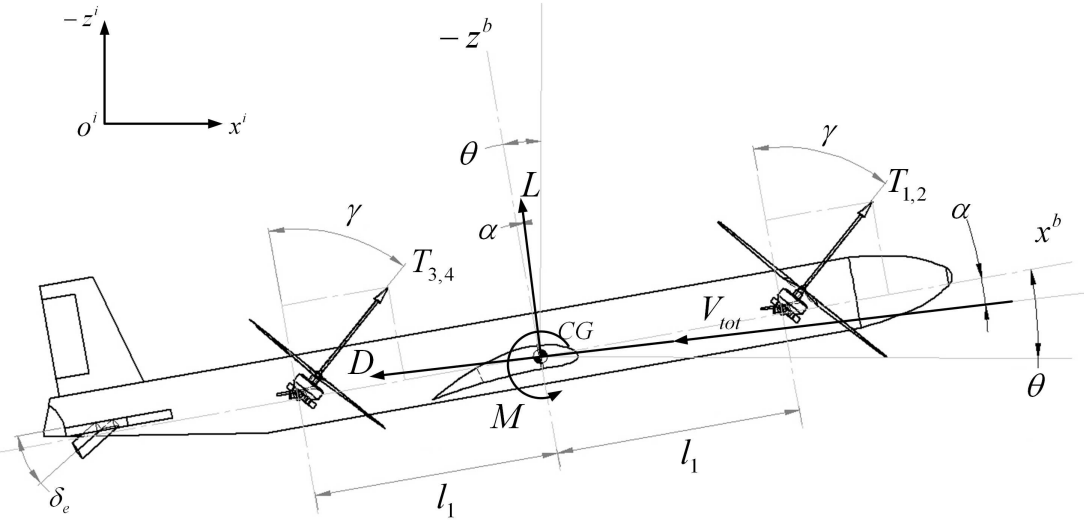

Figure 1.21: Free-body scheme showing the forces acting on the Quad-tilting MAV. 
$-\mathcal{F}^{b}$ denotes the body-fixed frame, with origin, $O^{b}$, at the center of gravity $C G$. This frame is associated to the vector basis $\left\{\boldsymbol{i}_{\boldsymbol{b}}, \boldsymbol{j}_{\boldsymbol{b}}, \boldsymbol{k}_{\boldsymbol{b}}\right\}$.

$-\mathcal{F}^{a}$ denotes the aerodynamic frame, with origin, $O^{b}$, at the center of gravity $C G$. This frame is associated to the vector basis $\left\{\boldsymbol{i}_{\boldsymbol{a}}, \boldsymbol{j}_{\boldsymbol{a}}, \boldsymbol{k}_{\boldsymbol{a}}\right\}$.

- The orthonormal transformation matrices $\mathcal{R}^{b i}$ and $\mathcal{R}^{a b}$, respectively used to transform a vector from $\mathcal{F}^{b} \rightarrow \mathcal{F}^{i}$ and $\mathcal{F}^{a} \rightarrow \mathcal{F}^{b}$ within the longitudinal plane (pitch axis), are given by:

$$
\mathcal{R}^{b i}=\left(\begin{array}{ccc}
\cos \theta & 0 & \sin \theta \\
0 & 1 & 0 \\
-\sin \theta & 0 & \cos \theta
\end{array}\right), \mathcal{R}^{a b}=\left(\begin{array}{ccc}
\cos \alpha & 0 & \sin \alpha \\
0 & 1 & 0 \\
-\sin \alpha & 0 & \cos \alpha
\end{array}\right)
$$

\section{Aerodynamics}

It is important to consider these forces properly because they are fundamentally affected by the vehicle's motion and thus they alter the basic dynamics involved. The analysis used in the present paper will be based on a combination of a low-order panel method aerodynamic model coupled with a simple actuator disc model of the flow induced by the propellers. In order to proceed with the aerodynamic analysis, it is worth to mention the following assumptions:

- A1. The vehicle is a rigid body, i.e. the felexibility of the aircraft wings or fuselage will be neglected.

- A2. Non-varying mass is considered $(\dot{m}(t)=0)$.

- A3. The aerodynamic center $(A C)$ and the center of gravity $(C G)$ are coincident.

In order to determine the aerodynamic forces exerted on the vehicle, we need to know both the direction and velocity of the total airflow vector. We can identify three wind vectors acting on the vehicle: the airflow speed $\boldsymbol{V}_{\boldsymbol{p}}$ produced by the rotors, the $V_{b}$ airflow generated by the translational motion of the body $(U, W)$ and a third component due to the external wind (disturbance) $V_{e}$, generally unknown. Hence, the total wind vector in the body frame can be written as

$$
\boldsymbol{V}_{\text {tot }}=\boldsymbol{V}_{\boldsymbol{p}}(\gamma)+\boldsymbol{V}_{\boldsymbol{b}}+\boldsymbol{V}_{\boldsymbol{e}}
$$

where $\boldsymbol{V}_{\text {tot }}=\left(v_{u}, v_{w}\right)^{T}$. The total wind vector $\boldsymbol{V}_{\boldsymbol{t} \text { to }}$ experienced by the wing varies depending on the flight mode. Within the $H F$ and $S F F$ regimes the wing is not washed by the propeller airflow $V_{p}$ (Fig. ??), while in the $F F F$ mode, it is assumed that the wing is significatively submerged (Fig. ??) by $\boldsymbol{V}_{\boldsymbol{p}}$. Therefore the propeller slipstream $\boldsymbol{V}_{\boldsymbol{p}}$ is disregarded in $H F$ and $S F F$. To include the behavior of $\boldsymbol{V}_{\boldsymbol{p}}$ in the equations let us introduce the following function

$$
\xi(\gamma)= \begin{cases}0 & \text { if } \gamma \leq \frac{\pi}{3} \\ 1 & \text { if } \gamma>\frac{\pi}{3}\end{cases}
$$




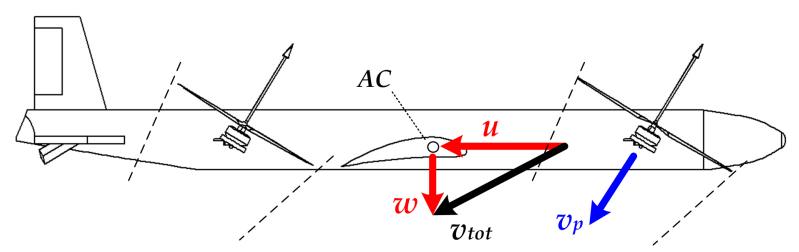

Figure 1.22: Airflow profile generated by the rotors during the flight envelope. Relative wind velocity in HF and SFF modes.

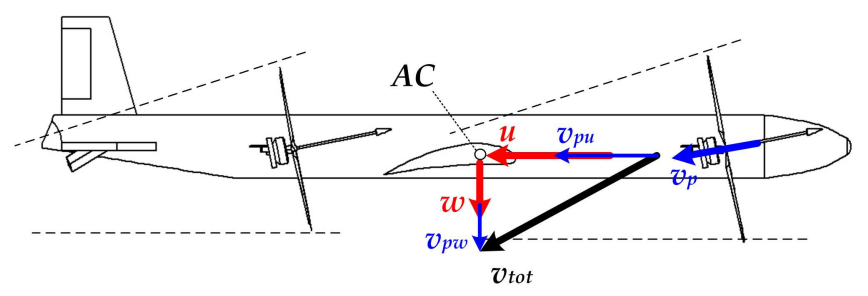

Figure 1.23: Relative wind velocity in FFF mode.

The parallel wind velocity $v_{u}$ and the normal wind velocity $v_{w}$ components at the wing encompass the velocity that the vehicle experiments through the air and the corresponding components of $\boldsymbol{V}_{\boldsymbol{p}}$ due the tilting of the rotors and the aleatory external wind $\boldsymbol{V}_{\boldsymbol{e}}$, i.e.

$$
\begin{aligned}
\boldsymbol{V}_{\boldsymbol{u}} & =\left(u+\xi(\gamma) v_{p} \sin (\gamma)+v_{e_{u}}\right) \boldsymbol{i}_{\boldsymbol{b}} \\
\boldsymbol{V}_{\boldsymbol{w}} & =\left(w+\xi(\gamma) v_{p} \cos (\gamma)+v_{e_{w}}\right) \boldsymbol{k}_{\boldsymbol{b}}
\end{aligned}
$$

Assuming purely axial flow into the propellers, simple actuator disc theory [STE 04] gives the induced propeller velocity for the $i^{\text {th }}$ rotor as

$$
v_{p_{i}}=\sqrt{\frac{2 T_{i}}{\rho A_{p}}}
$$


where $A_{p}$ is the total disc-area of the propeller and $\rho$ the air density. Figure ?? shows the aerodynamic forces on a small UAV with a tilt angle $\gamma$. The forces consist of a lift force, $L$, perpendicular to the total flow vector, $\boldsymbol{V}_{t o t}$, a drag force $D$ parallel to $\boldsymbol{V}_{\boldsymbol{t} \text { tot }}$, and the airfoil's pitching moment, $M$, about the positive cartesian $y$-axis. The above discussion can be summarized by:

$$
\begin{aligned}
& C_{l}=C_{l_{\alpha}} \alpha \\
& C_{d}=C_{d_{p}}+C_{d_{i}} \\
& C_{m}=C_{m_{\alpha}} \alpha
\end{aligned}
$$

where these equations are standard aerodynamic non-dimensional lift, drag and moment coefficients ${ }^{1}$ To obtain the lift and drag forces and the pitching moment on the aircraft it is only necessary to obtain the total wind velocity vector $\boldsymbol{V}_{\text {tot }}$, see (1.122), the angle of attack and the aerodynamic parameters $C_{l_{\alpha}}, C_{l_{\delta}}, C_{d}, C_{m_{\delta}}, C_{m_{\alpha}}$ which depend on the geometry of the vehicle.

$$
\begin{aligned}
& L=\frac{1}{2} C_{l} \rho V_{t o t}^{2} S \\
& D=\frac{1}{2} C_{d} \rho V_{t o t}^{2} S \\
& M=\frac{1}{2} C_{m} \rho V_{t o t}^{2} S \bar{c}
\end{aligned}
$$

In these equations $S$ and $\bar{c}$ are the area and the wing chord respectively. The angle of attack $\alpha$ and the magnitude of $\boldsymbol{V}_{\text {tot }}$ are obtained through the following equations

$$
\begin{aligned}
& \alpha=\arctan \left(v_{w} / v_{u}\right) \\
& \left|\boldsymbol{V}_{\text {tot }}\right|=\sqrt{v_{w}^{2}+v_{u}^{2}}
\end{aligned}
$$

The lift force will depend on the velocity $\boldsymbol{V}_{\boldsymbol{t} \text { tot }}$ and the angle of attack. The figure ?? represents the different values of lift for several speed conditions:

\section{Forces exerted on the Quad-plane}

The vector that contains the set of forces applied to the Quad-plane (Fig. ?? ) is given by

$$
m \ddot{\xi}=\mathcal{R}^{b i} T^{b}+\mathcal{R}^{b i} \mathcal{R}^{a b} A^{a}+\mathcal{W}^{i}
$$

where, $\boldsymbol{\xi}=(x, y, z)^{T}$ is the $C G$ 's position vector in $\mathcal{F}^{i}, \boldsymbol{T}^{\boldsymbol{b}}=(0,0,-T)^{T}$ is the collective thrust in $\mathcal{F}^{b}, \boldsymbol{A}^{\boldsymbol{a}}=(-D, 0,-L)^{T}$ is the vector of aerodynamic forces in $\mathcal{F}^{a}$ and finally $\mathcal{W}^{i}=(0,0, m g)^{T}$ denotes the weight of the vehicle in $\mathcal{F}^{i}$. The four propellers produce the collective thrust $T$, which can be modeled as

$$
T=K_{l} \sum_{i=1}^{i=4} \omega_{i}^{2}
$$

1. $C_{*}$ slopes are obtained from the software XFOIL. 


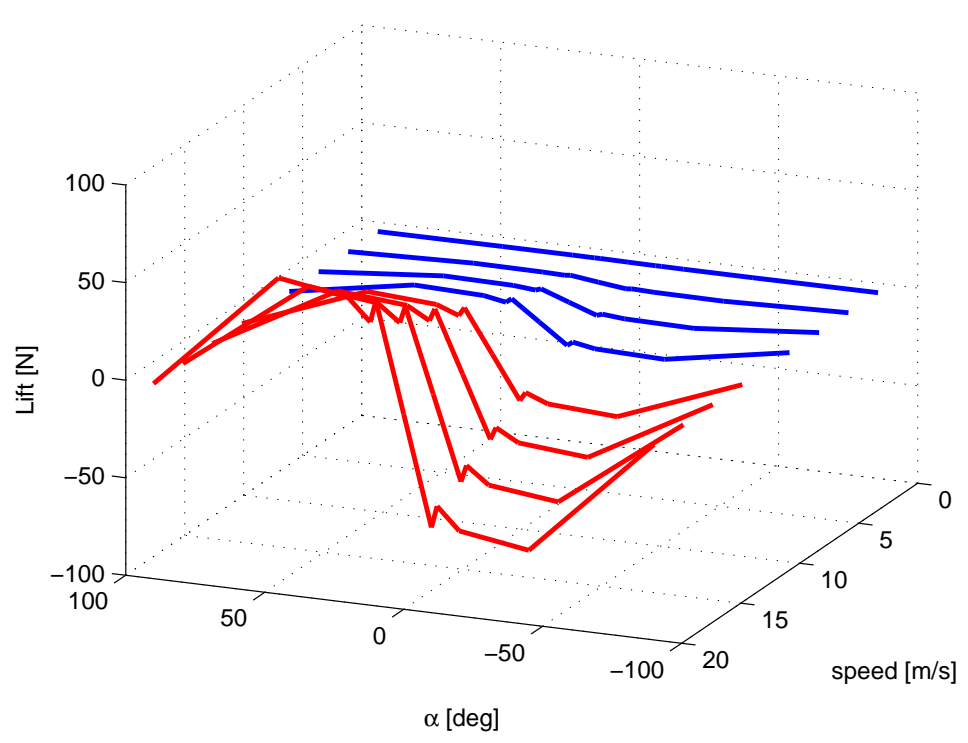

Figure 1.24: Lift values for different velocities.

where $\omega_{i}$ is the angular velocity of $i^{t h}$-rotor, $K_{l}$ is a lift factor depending on the aerodynamic parameters of the propeller. Note that the vector of aerodynamic forces $A^{a}$ is not only involved in translational motion, but also in the rotational motion of the vehicle, as is shown next.

\section{Moments acting on the Quad-plane}

The forces shifted away from the center of gravity $C G$ induce moments causing the rotational motion. The corresponding vectorial equation grouping the moments exerted about $C G$ is written as

$$
\tau^{b}=\tau_{T}^{b}+\tau_{M}^{b}+\tau_{G}^{b}
$$

where $\boldsymbol{\tau}_{\boldsymbol{T}}$ is the induced moment due the difference of thrust between $T_{3,4}$ and $T_{1,2}$, $\tau_{M}$ is the airfoil's pitching moment, $\tau_{G}$ is the gyroscopic moment. $\tau_{T}$ is obtained through

$$
\boldsymbol{\tau}_{\boldsymbol{T}}^{\boldsymbol{b}}=l_{1}\left(-T_{3,4} \cos \gamma+T_{1,2} \cos \gamma\right) \boldsymbol{j}_{\boldsymbol{b}}
$$

where, $l_{1}$ is the distance from the $C G$ to the rotors. The airfoil's pitching moment $\tau_{M}$ 
is obtained from the airfoil's $C_{m}$ slope and the lift contribution of the elevator.

$$
\tau_{M}^{b}=M j_{b}
$$

The gyroscopic moment $\tau_{G}$ arises from the combination of the airframe's angular speed $\boldsymbol{\Omega}^{b}=(p, q, r)^{T}$ and the rotors angular speed $\omega_{i}$. The $\tau_{G}$ vector is then modeled as $\sum_{i=1}^{4}\left(\boldsymbol{\Omega}^{b} \times I_{p} \boldsymbol{\omega}_{\boldsymbol{i}}\right)$, leading to

$$
\boldsymbol{\tau}_{\boldsymbol{G}}^{\boldsymbol{b}}=I_{p}\left[q\left(\omega_{2}-\omega_{1}+\omega_{3}-\omega_{4}\right) \boldsymbol{i}_{\boldsymbol{b}}+p\left(\omega_{1}-\omega_{2}-\omega_{3}+\omega_{4}\right) \boldsymbol{j}_{\boldsymbol{b}}\right]
$$

where, $I_{p}$ represents the inertia moment of the propeller. For simplicity we do not take into account the drag torque due to the propeller drag force. Since the present paper concentrates on the longitudinal flight of the vehicle, the corresponding scalar equations modeling the forces and moments applied to the vehicle are written as:

$$
\begin{aligned}
& m \ddot{X}=-T_{3,4} \sin (\theta+\gamma)-T_{1,2} \sin (\theta+\gamma)-L \sin (\theta-\alpha)-D \cos (\theta-\alpha) \\
& m \ddot{Z}=D \sin (\theta-\alpha)-T_{1,2} \cos (\theta+\gamma)-L \cos (\theta-\alpha)-T_{3,4} \cos (\theta+\gamma)+g \\
& I_{y y} \ddot{\theta}=M+l_{1}\left(-T_{3,4} \cos \gamma+T_{1,2} \cos \gamma\right)+I_{p} p\left(\omega_{1}-\omega_{2}-\omega_{3}+\omega_{4}\right)
\end{aligned}
$$

\section{FFF mathematical model}

In this regime the vehicle essentially behaves as an airplane, thus we can consider the common longitudinal aircraft model [STE 92]. In addition to the body-axis equations, it is important to express the equations of motion in the wind axis, because the aerodynamic forces act in these axis and the magnitude of $\boldsymbol{V}_{\boldsymbol{t} o t}$ (written as $V$ from here onwards"), $\alpha$ can be expressed in terms of $u$ and $w$. This reference system is used for translational equations because angle of attack and velocity are either directly measurable or closed related to directly measurable quantities, while the body axis velocities $(u, w)$ are not. The equations of motion take the form

$$
\begin{aligned}
\dot{V} & =\frac{1}{m}\left[-D+T_{t} \cos \alpha-m g(\cos \alpha \sin \theta-\sin \alpha \cos \theta)\right] \\
\dot{\alpha} & =\frac{1}{V m}\left[-L-T_{t} \sin \alpha+m g(\cos \alpha \cos \theta+\sin \alpha \sin \theta)\right]+q \\
\dot{\theta} & =q
\end{aligned}
$$

The angles $\theta$ and $\alpha$ lie in the same vertical plane above the north-east plane (Fig. ??), and their difference is the flight-path angle $\Gamma=\theta-\alpha$ (Fig. ??). Under this definition 


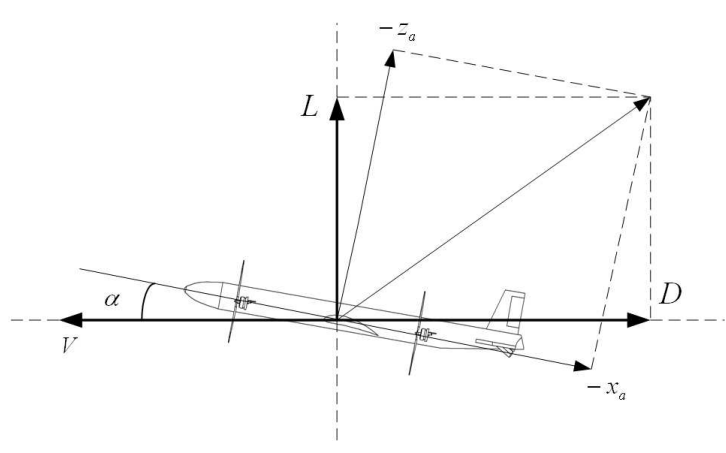

Figure 1.25: Wind-Axis

Reference Frame.

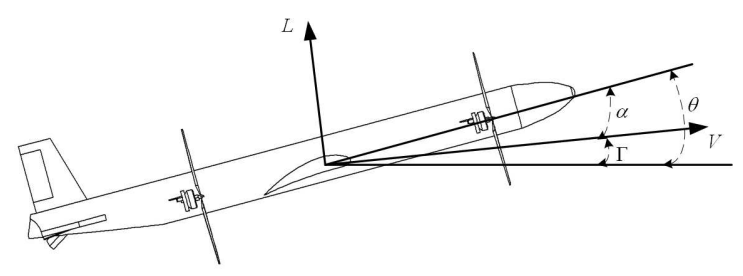

Figure 1.26: Flight-path angle definition.

and from the last equation of (1.135) we obtain the next mathematical model

$$
\begin{aligned}
\dot{V} & =\frac{1}{m}\left[-D+T_{t} \cos \alpha-m g \sin \Gamma\right] \\
\dot{\alpha} & =\frac{1}{V m}\left[-L-T_{t} \sin \alpha+m g \cos \Gamma\right]+q \\
\dot{\theta} & =q \\
\dot{q} & =\frac{1}{I_{y y}} M
\end{aligned}
$$

\subsubsection{Transition}

The flight envelope of the vehicle encompasses different flight conditions, achieved by means of the collective angular displacement of the rotors. Indeed, is this 


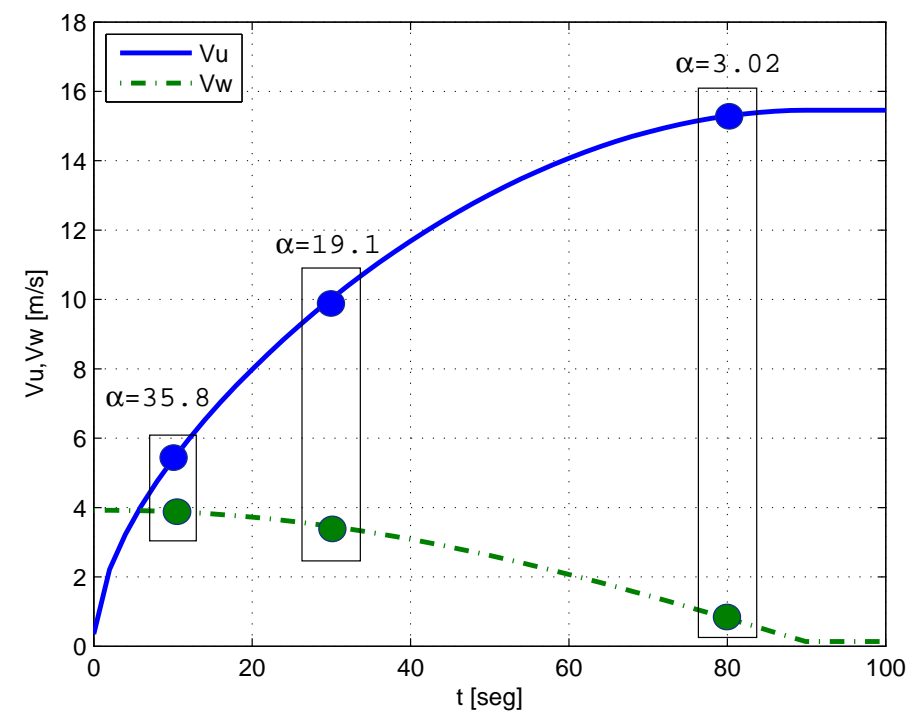

Figure 1.27: Behavior velocities during the tilting of the rotors.

tilting that provides a continuous mechanism to perform the operational transition. To illustrates this, let us consider the following scenario:

$-T_{t} \geq m g$ i.e. the vehicle flies at a stabilized altitude.

$-\theta \approx 0$ i.e. stabilized vertical flight.

Is clear that as $\gamma$ is tilted the horizontal velocity increases, while the vertical velocity is reduced (figures ?? and ??). These facts affect proportionally to the forces coming from the rotors and the wing.

Thus, both vertical and horizontal controllers can still be used at the same time whose actions is controlled by $\gamma$. The vertical collective thrust is gradually reduced inhibiting the action of vertical controller and allowing the action of the horizontal controller and viceversa. So, for example, for larger values of $\gamma$, i.e. $\gamma>45$, the rotorcraft behaves more like a classical airplane. As the vehicle is gaining speed due to rotors tilt $(\gamma)$, then aerodynamic forces arise. For this reason we consider that the control of vertical and forward flight are active during the whole flight envelope. 


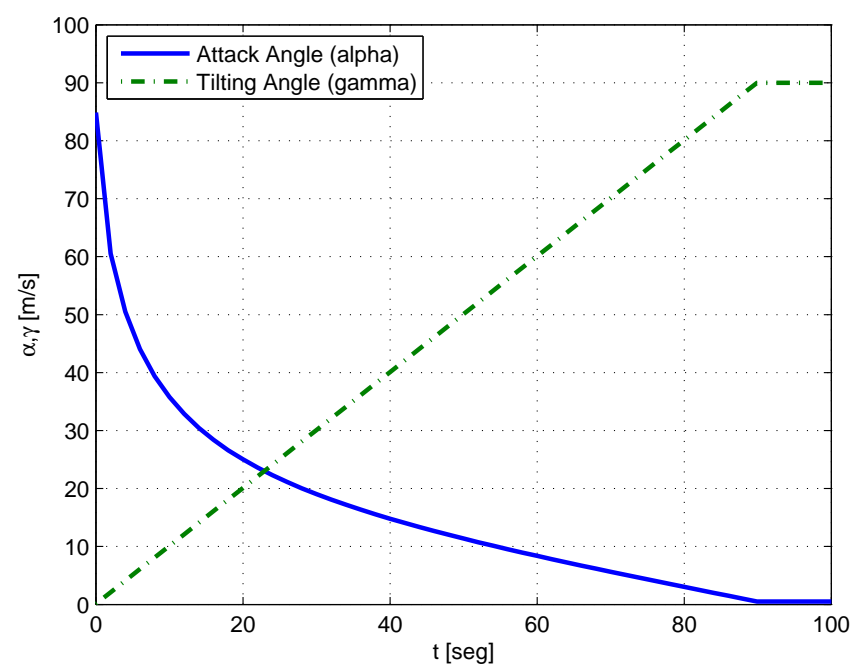

Figure 1.28: Behavior of angles $\gamma$ and $\alpha$ during the tilting of the rotors.

\subsubsection{Control strategy for hover flight mode}

The vertical flight of the Quad-plane represents a challenging stage due to the aircraft's vertical dynamics are naturally unstable. In this regime, the Quad-plane aerial robot aims to emulate the flight behavior of a Quadrotor which features and non conventional Quadrotor design, i.e. an asymmetrical H-form structure.

Vertical flight regime encompasses two dynamic subsystems: the altitude dynamics, actuated by the thrust $T$, and the horizontal translational motion, generated by the pitch attitude. Taking into account the item 1) presented in section II, we can consider a simplified model from which is derived the controller in $\mathrm{HF}$ regime (i.e. $\alpha \approx 0$ since $\gamma=0)$.

For simplicity we consider that the gyroscopic moment is very small. These considerations allows us to rewrite (1.135) as

$$
\begin{aligned}
& \ddot{X}=-\left(\frac{T_{3,4}+T_{1,2}}{m}\right)(\sin \theta) \\
& \ddot{Z}=-\left(\frac{T_{3,4}+T_{1,2}}{m}\right)(\cos \theta)+g \\
& \ddot{\theta}=-\left(\frac{l_{1}}{I_{y y}}\right)\left(-T_{3,4}+T_{1,2}\right)
\end{aligned}
$$


If we rename the total thrust as $T_{t}=T_{3,4}+T_{1,2}$, and the difference of these thrusts as $T_{d}=T_{1,2}-T_{3,4}$. Then

$$
\begin{aligned}
\ddot{X} & =-\frac{T_{t}}{m} \sin \theta \\
\ddot{Z} & =-\frac{T_{t}}{m} \cos \theta+g \\
\ddot{\theta} & =-\frac{l_{1}}{I_{y y}} T_{d}
\end{aligned}
$$

thus, we have derived a simple model, suitable for controller design. The altitude (1.139b) can be stabilized via a feedback-linearizable input through the total thrust $T_{t}$

$$
T_{t}=-\frac{m u_{z}-m g}{\cos (\theta)}
$$

where $u_{z}=-k_{p_{z}}\left(z-z^{d}\right)-k_{d_{z}} \dot{z}$ with $k_{p_{z}}, k_{d_{z}}>0$ and $z_{d}$ is the desired altitude. Since the vehicle works in an area close to $\theta \approx 0$, the singularity is avoided. For the subsystem 1.139a and $1.139 \mathrm{c}$, a two-level hierarchical control scheme is used to stabilize its dynamics. The outer-loop control stabilizes the translational motion (slow dynamics [KOK 86]) along the $x$-axis, while the inner-loop control stabilizes the attitude (fast dynamics). Introducing (1.140) into (1.139a) and assuming that $z \approx z^{d}$, namely $u_{z} \rightarrow 0$, leads to

$$
\ddot{x} \approx-g \tan \theta=-g \tan u_{x}
$$

For the horizontal motion (1.141), $\theta$ can be considered as virtual control input $u_{x}$. However, it is a state not an actual control. Given that $\dot{\theta}^{d}$ is slowly time-varying, we will assume that the $x$-dynamics converges slower than the $\theta$-dynamics. The reference for the inner-loop systems is

$$
\begin{aligned}
& u_{x}=\theta^{d}=\arctan \left(\frac{-v_{x}}{g}\right) \\
& \dot{u}_{x}=\dot{\theta}^{d} \approx 0
\end{aligned}
$$

where $v_{x}=k_{v_{x}} \dot{x}+k_{p_{x}} x$ with $k_{v_{x}}, k_{p_{x}}>0$. Using the linearizing control input (1.142) in (1.141) yields

$$
\ddot{x}=v_{x}, \text { provided that } \tilde{\theta}=0 \text { (i.e. } \theta=\theta^{d} \text { ) }
$$

As the previous equation shows, the success of the outer-loop controller relies directly on the inner-loop attitude control performance, thus the inner loop controller must guarantee the stabilization of the attitude around the reference. For this reason, the stability analysis of the inner-loop controller is presented next. Consider the following positive function which is an unbounded function

$$
V(\tilde{\theta}, \dot{\theta})=\frac{1}{2} I_{y y} \dot{\theta}^{2}+\ln (\cosh \tilde{\theta})
$$


Using (1.139) its corresponding time-derivative yields

$$
\dot{V}(\tilde{\theta}, \dot{\theta})=I_{y y} \dot{\theta}\left(-\frac{l_{1}}{I_{y y}} T_{d}\right)+\dot{\tilde{\theta}} \tanh \tilde{\theta}
$$

Considering $\dot{\tilde{\theta}}=\dot{\theta}$, thus (1.145) may be rewritten as

$$
\dot{V}(\tilde{\theta}, \dot{\theta})=\dot{\theta}\left(-l_{1} T_{d}+\tanh \tilde{\theta}\right)
$$

Using the control input

$$
T_{d}=\frac{\tanh \tilde{\theta}+\tanh \dot{\theta}}{l_{1}}
$$

in (1.146) yields

$$
\dot{V}(\tilde{\theta}, \dot{\theta})=-\dot{\theta} \tanh \dot{\theta}
$$

where $\dot{V}(\tilde{\theta}, \dot{\theta}) \leq 0$. Therefore, the origin $(\tilde{\theta}, \dot{\theta})$ is stable and the state vector remains bounded. The asymptotic stability analysis can be obtained from LaSalle's Theorem. Therefore, $\tilde{\theta} \rightarrow 0$ and $\dot{\theta} \rightarrow 0$ as $t \rightarrow \infty$.

\subsubsection{Control strategy for forward flight mode}

In this section the flight path angle $\Gamma$ will be controlled using the backstepping algorithm taking the following approximations into consideration:

- The air speed is assumed constant, $\dot{V}=0$ [MAT 07].

- From the definition of flight-path angle, the dynamics $\dot{\Gamma}=\dot{\theta}-\dot{\alpha}$ yields $\dot{\Gamma}=$ $\frac{1}{m V}\left[T_{t} \sin \alpha+L-m g \cos \Gamma\right]$.

- The thrust term $T_{t} \sin \alpha$ in (1.137) will be neglected as it is generally much smaller than lift.

$-C_{m}=C_{m_{\delta}}(\alpha) \delta$, since the main contribution to $M$ is provided by the elevator.

With these considerations in mind and using the change of coordinates $\zeta=\Gamma-$ $1 / 2 \pi$, the system (1.137) may be expressed as

$$
\begin{aligned}
\dot{\zeta} & =-\frac{g \cos (z+1 / 2 \pi)}{V}+\frac{C_{l_{\alpha}} \alpha}{m V} \\
\dot{\alpha} & =\frac{g \cos (z+1 / 2 \pi)}{V}-\frac{C_{l_{\alpha}} \alpha}{m V}+q \\
\dot{q} & =\frac{1}{I_{y y}} C_{m_{\delta}} \delta
\end{aligned}
$$


Equation (1.150) is now in feed forward form for backstepping procedure. For notational simplification, let

$$
\begin{aligned}
\dot{x} & =f(x)+\xi_{1} \\
\dot{\xi}_{1} & =f_{1}\left(x, \xi_{1}\right)+\xi_{2} \\
\dot{\xi}_{2} & =f_{2}+g_{2}\left(\xi_{1}\right) u
\end{aligned}
$$

with

$$
\begin{aligned}
& x=\frac{m V \Gamma}{C_{l_{\alpha}}} ; \\
& f(x)=-\frac{g}{V} \cos \left(\frac{C_{l_{\alpha}} x}{m V}\right) \\
& \xi_{1}=\alpha \text {; } \\
& \xi_{2}=q ; \\
& u=\delta \text {; } \\
& \begin{array}{ccc}
f_{1}\left(x, \xi_{1}\right) & = & \frac{g}{V} \cos \left(\frac{C_{l_{\alpha}} x}{m V}\right)-\frac{C_{l_{\alpha}} \xi_{1}}{m V} \\
f_{2} & = & 0 \\
g_{2}\left(\xi_{1}\right) & = & \frac{1}{J_{y}} C_{m_{\delta}}
\end{array}
\end{aligned}
$$

Defining the following error states as

$$
\begin{aligned}
& e \triangleq x-x_{\text {des }} \\
& e_{1} \triangleq \xi_{1}-\xi_{1, \text { des }} \\
& e_{2} \triangleq \xi_{2}-\xi_{2, \text { des }}
\end{aligned}
$$

Now, following the backstepping procedure differentiating the first equation in (1.152) yields

$$
\dot{e}=f(x)+\xi_{1, \text { des }}+e_{1}-\dot{x}_{d e s}
$$

where $\xi_{1, \text { des }}$ is viewed as a virtual control for the last equation, choosing as $\xi_{1, d e s}=$ $-f(x)-k_{1} e+\dot{x}_{d e s}$. Then substituting this virtual control in (1.153) we have that

$$
\dot{e}=-k e+e_{1}
$$

Repeating the same procedure, differentiating $e_{1}$ yields

$$
\dot{e}_{1}=f_{1}\left(x, \xi_{1}\right)+e_{2}+\xi_{2, \text { des }}-\dot{\xi}_{1, \text { des }}
$$

Let $\xi_{2, \text { des }}=-f_{1}\left(x, \xi_{1}\right)-e-k_{1} e_{1}+\dot{\xi}_{1, \text { des }}$ so that

$$
\dot{e}_{1}=-e-k_{1} e_{1}+e_{2}
$$

As a last step, now the real control signal is obtained in similar way. Differentiating $e_{2}$ yields

$$
\dot{e}_{2}=f_{2}+g_{2}\left(\xi_{1}\right) u-\dot{\xi}_{2, d e s}
$$


Let

$$
u=\frac{1}{g_{2}\left(\xi_{1}\right)}\left[-f_{2}-e_{1}-k_{2} e_{2}+\dot{\xi}_{2, d e s}\right]=u\left(\dddot{z}_{d}, \ddot{z}_{d}, \dot{z}_{d}, z_{d}, e, e_{1}, e_{2}\right)
$$

so that

$$
\dot{e}_{2}=-e_{1}-k_{2} e_{2}
$$

It is important to ensure that $g_{2}\left(\xi_{1}\right) \neq 0$, which occurs only with big enough
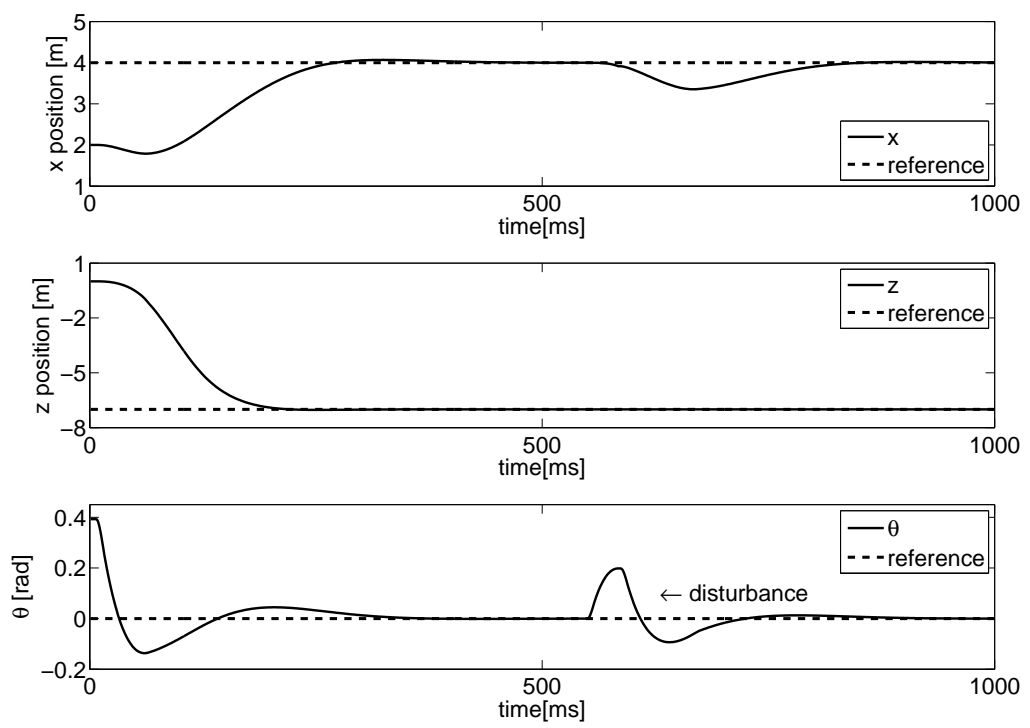

Figure 1.29: Position and attitude of the vehicle under disturbance condition.

negative values of $\alpha$. These values are assumed to be impossible to achieve in standard operation of the airplane, so avoiding division by zero. Equations $(1.154,1.156,1.159)$ expressed in vectorial form

$$
\dot{e}=-K e+S e
$$

$S=-S^{T}$ satisfies $e^{T} S e=0, \forall e$, so that with the Lyapunov-candidate-function $V(\boldsymbol{e})=\frac{1}{2} \boldsymbol{e}^{T} \boldsymbol{e}$, and the time derivative evaluated in the trajectories yields

$$
\dot{V}(\boldsymbol{e})=\boldsymbol{e}^{T}(-K \boldsymbol{e}+S \boldsymbol{e})=-\boldsymbol{e}^{T} K \boldsymbol{e}<0, \forall \boldsymbol{e}
$$

This proves that the above differential equation, is asymptotically stable about the origin. 

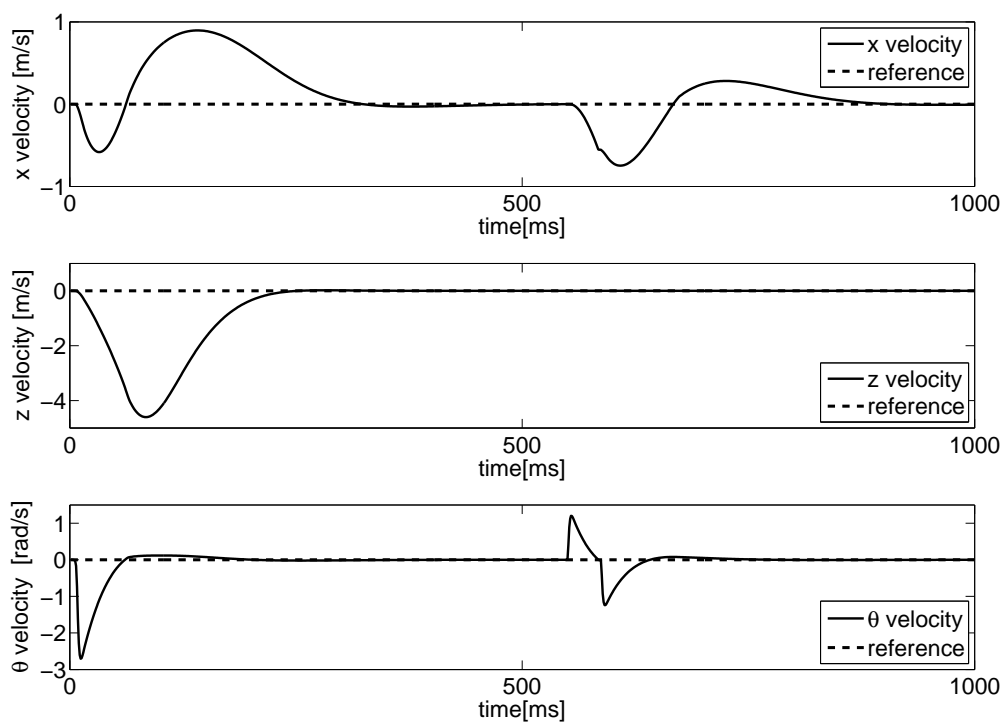

Figure 1.30: Derivatives of the position and attitude of the vehicle under disturbance condition.

\subsubsection{Simulation results}

\section{HF}

The performance of the nonlinear controller presented in the previous section is evaluated on the dynamic model (1.139) in MATLAB/Simulink. We started the Quadplane at the position $\left(x_{0}, z_{0}, \theta_{0}\right)=\left(2,0, \frac{\pi}{8}\right)$ and $(\dot{x}, \dot{z}, \dot{\theta})=(0,0,0)$. The aircraft had the task of performing hover flight at $\left(x^{d}, z^{d}, \theta^{d}\right)=(4,-7,0)$. Figures ?? and ?? show the evolution and convergence of the states $(x, \dot{x}, z, \dot{z}, \theta, \dot{\theta})$ to the desired references with the initial conditions mentioned above. It is important to note that position and angle references are tracked with negligible steady state errors. The controller is robust in the presence of a perturbation in $t=600 \mathrm{~ms}$ with a magnitude of $1 / 8 \pi$ radians, as seen in figures ?? and ??.

The control inputs are depicted in the figure ??, which shows the reaction to the disturbance. 

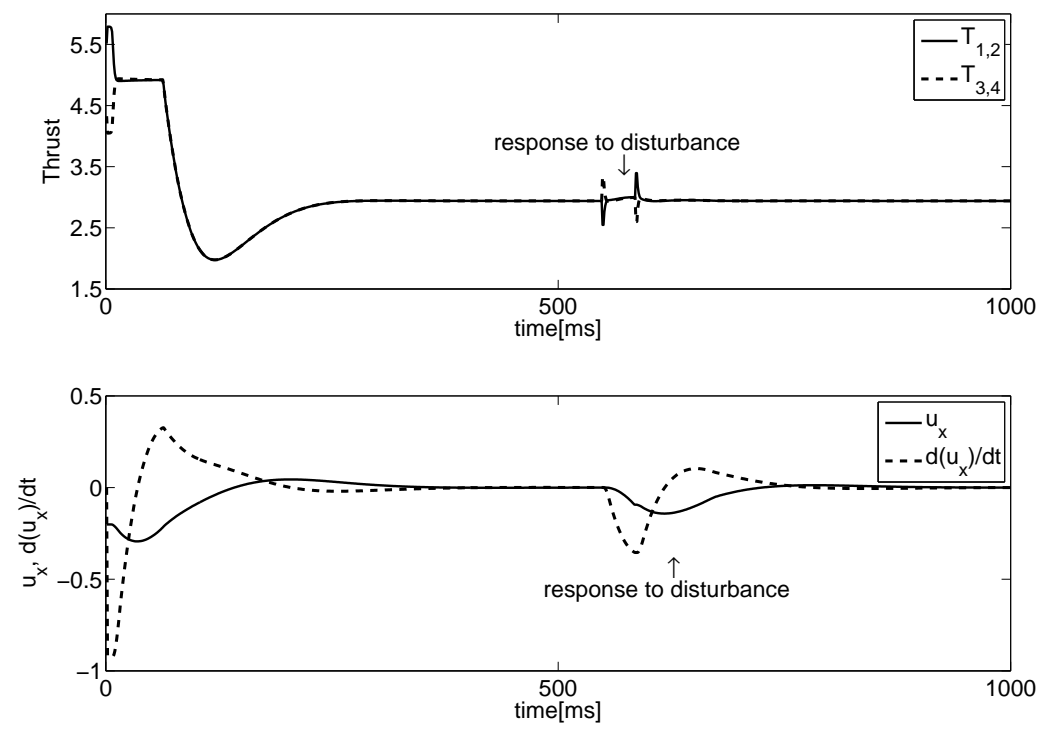

Figure 1.31: UAV's control inputs and response to disturbance.

\section{FFF}

After the vehicle experiments the transition-flight, its behavior is like an airplane. We have considered the next initial conditions for purposes of simulation: $\Gamma_{0}=5$, $\alpha_{0}=5$ and $\theta_{0}=10$. The aircraft had the task of tracking a trajectory shown in the first part of Fig. ??. This figure shows the evolution and tracking trajectory of the states $(\Gamma, \alpha, \theta)$ to the desired reference, with the initial conditions mentioned above. It is important to note that angle references are tracked with negligible steady state errors.

\subsection{Concluding remarks}

In this Chapter Euler-Lagrange approach has been applied for obtaining a simplified model of a quad-rotor rotorcraft.

The longitudinal dynamics of this aircraft including its aerodynamics are derived at the hover and forward flight operating mode. The proposed control strategies were evaluated, at simulation level, for the nonlinear dynamic model, obtaining satisfactory 

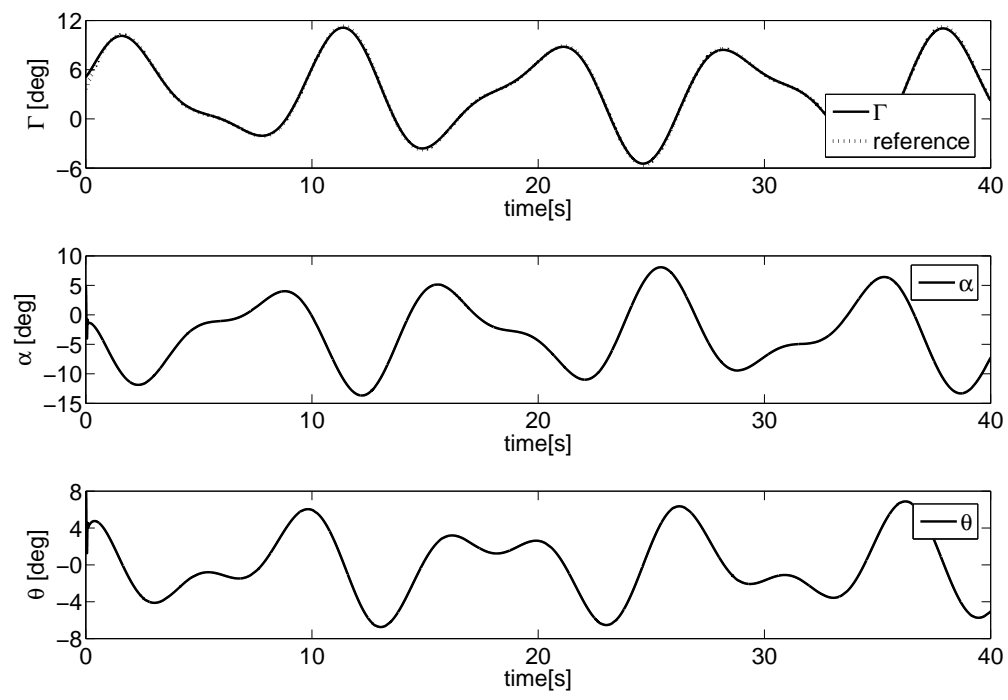

Figure 1.32: Tra-

jectory tracking.

results. The proposed control algorithm is based on an inner-outer loop scheme since it is suitable for implementation purposes.

For energy-saving purposes during forward flight (airplane mode), it is proposed that the vehicles can lead their orientation towards wind velocity vector. To achieve this objective, the vehicles could easily rotate their orientation (yaw movement in helicopter mode) and once addressed the wind vector, switch to airplane mode. In the Quad-plane configuration, this process could be quite simple, since the vehicle is always maintained with the roll and pitch angles close to zero, which is not possible in the case of a tail-sitter configuration since within vertical mode the wing surface is highly vulnerable to wind gusts.

\subsection{Bibliography}

[AND 90] Anderson B., Moore J., Optimal Control, Prentice Hall, 1990.

[BAR 94] BARMish B., New Tools for Robustness of Linear Systems, Macmillan, 1994.

[BLY 06] BLYENBURG P., "UAVs systems, the global perspective”, UVS International, 2006.

[CAS 05] Castillo P., Lozano R., Dzul A., Modelling and Control of Mini-Flying Machines, Springer-Verlag, 2005. 

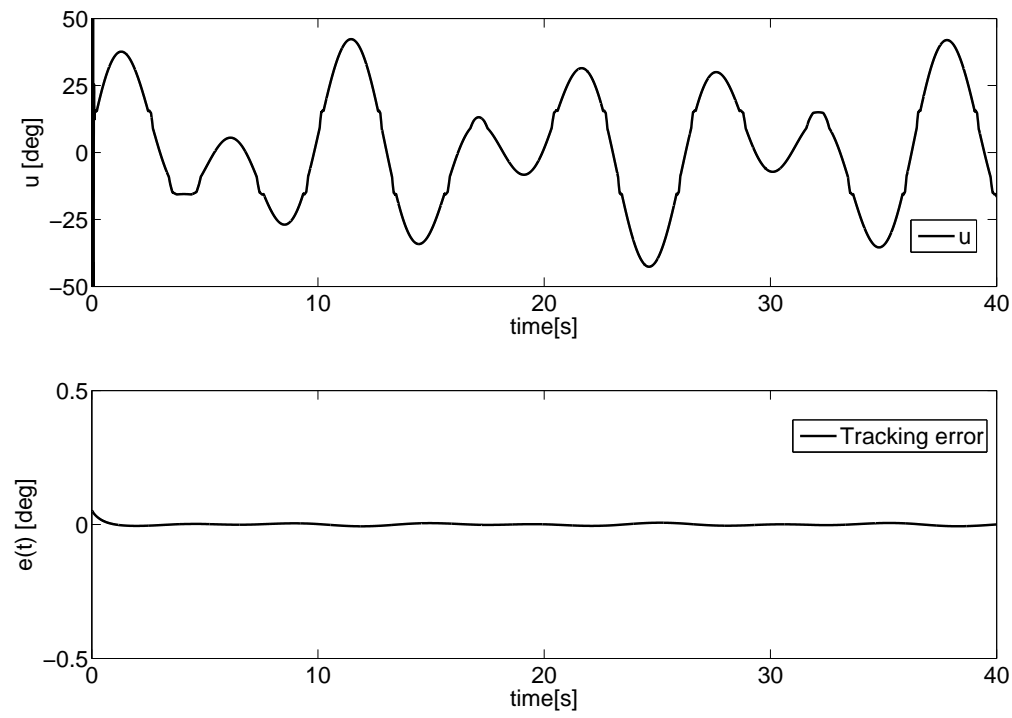

Figure 1.33: Control and error defined by $e=\Gamma-\Gamma_{d}$.

[ESC 06] Escareno J., SAlazar S., Lozano R., "Modeling and Control of a Convertible VTOL Aircraft", 45th IEEE Conference on Decision and Control, 2006.

[ESC 07] Escareno J., Stone H., Sanchez A., Lozano R., "Modeling and Control Strategyfor the transition of a Convertible UAV", European Control Conference (ECC07), 2007.

[ETK 96] ETKIn B., REID L., Dynamics of Flight Stability and Control, John Wiley and Sons, 1996.

[GOl 83] Goldstein H., Poole C., Safko J., Classical Mechanics, Addison-Wesley, 1983.

[GRE 06] Green W., OH P., "Autonomous Hovering of a Fixed-Wing Micro Air Vehicle”, International Conference on Robotics and Automation, 2006.

[HEP 06] HePperle M., Aerodynamics for model aircraft, Technical report, 2006.

[KAN 01] KANNAN N., SEetharama M., "Longitudinal h1 stability augmentation system for a thrust-vectored unmanned aircraft", AIAA, Journal of Guidance, Control and Dynamics, vol. 28, 2001.

[KHA 02] KHALIL H., Nonlinear Systems, Prentice Hall, 2002.

[KOK 86] Kокотоvic P., O’ Reilly P., Khalil H., Singular Perturbation Methods in Control: Analysis and Design, Academic Press, 1986. 
[LEW 95] Lewis F., Syrmos V., Optimal Control, John Wiley and Sons, 1995.

[LIN 07] LiN F., Robust Control Design, An optimal control approach, John Wiley and Sons, 2007.

[MAT 07] Mates D., Hagstrom M., "Nonlinear Analysis and Synthesis Techniques for Aircraft Control", Lecture Notes in Control and Information Sciences, vol. 365, Springer, 2007.

[NON 07] NonAmi K., "Prospect and Recent Research and Development for Civil Use Autonomous Unmanned Aircraft as UAV and MAV”, Journal of System Design and Dynamics, vol. 1, 2007.

[ONE 08] Oner K., Cetinsoy E., Unel M., Aksit M., Kandemir I., Gulez K., "Dynamic Model and Control of a New Quadrotor Unmanned Aerial Vehicle with TiltWing Mechanism", vol. 45, World Academy of Science, Engineering and Technology, 2008.

[ROM 95] ROMERo G., COLlado J., "Robust stability of interval plants with perturbed time delay", Proceedings of the IEEE American Control Conference, 1995.

[ROM 97] Romero G., Analysis of Robust Stability of Dynamics Systems with Time Delay, $\mathrm{PhD}$ thesis, FIME - UANL, 1997.

[SNY 00] SNYDER D., “The Quad Tiltrotor: Its Beginning and Evolution”, Proceedings of the 56th Annual Forum, American Helicopter Society, 2000.

[SPO 01] SPOERRY, WONG K., "Design and developement of a micro air vehicle (mav)concept: Project bidule", 9th Australian International Aerospace Congress (AIAC), 2001.

[STE 92] Stevens B., LeWIS F., Aircraft Control and Simulation, John Wiley and Sons Inc, 1992.

[STE 04] Stengel R., Flight Dynamics, Princeton University Press, 2004.

[STO 02a] StOne H., "Aerodynamic modelling of a wing-in-slipstream tail-sitter UAV", AIAA Biennial International Powered Lift Conference and Exhibit, 2002.

[STO 02b] Stone H., "Aerodynamic Modeling of a Wing-in-Slipstream Tail-Sitter UAV", Biennial International Powered Lift Conference and Exhibit, 2002.

[STO 04] StOne H., "Control Architecture for a Tail-sitter Unmanned Air Vehicle", 5th Asian Control Conference, 2004. 